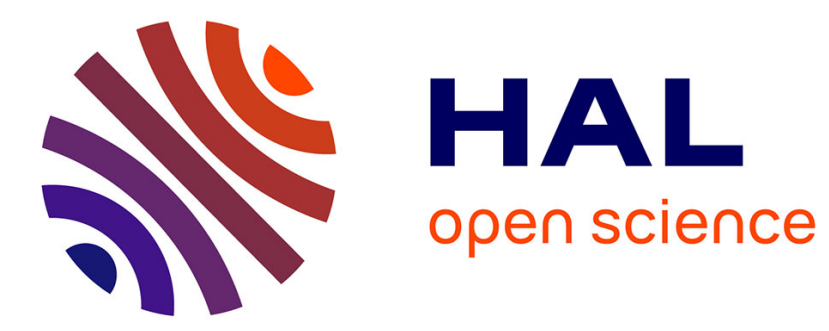

\title{
Dynamics of a small system coupled to a reservoir : reservoir fluctuations and self-reaction
}

\author{
J Dalibard, J. Dupont-Roc, C. Cohen-Tannoudji
}

\section{To cite this version:}

J Dalibard, J. Dupont-Roc, C. Cohen-Tannoudji. Dynamics of a small system coupled to a reservoir: reservoir fluctuations and self-reaction. Journal de Physique, 1984, 45 (4), pp.637-656. 10.1051/jphys:01984004504063700 . jpa-00209794

\section{HAL Id: jpa-00209794 https://hal.science/jpa-00209794}

Submitted on 1 Jan 1984

HAL is a multi-disciplinary open access archive for the deposit and dissemination of scientific research documents, whether they are published or not. The documents may come from teaching and research institutions in France or abroad, or from public or private research centers.
L'archive ouverte pluridisciplinaire HAL, est destinée au dépôt et à la diffusion de documents scientifiques de niveau recherche, publiés ou non, émanant des établissements d'enseignement et de recherche français ou étrangers, des laboratoires publics ou privés. 


\title{
LE JOURNAL DE PHYSIQUE
}

Classification

Physics Abstracts

$05.00-32.90$

\section{Dynamics of a small system coupled to a reservoir : reservoir fluctuations and self-reaction}

\author{
J. Dalibard, J. Dupont-Roc and C. Cohen-Tannoudji \\ Laboratoire de Spectroscopie Hertzienne de l'Ecole Normale Supérieure et Collège de France, \\ 24, rue Lhomond, 75231 Paris, France
}

(Reçu le 3 octobre 1983, accepté le 6 décembre 1983)

\begin{abstract}
Résumé. - Les processus physiques responsables de l'évolution d'un petit système $S$ faiblement couplé à un grand réservoir $\mathcal{R}$ sont analysés à partir des équations de Heisenberg. Les contributionṡ respectives des fluctuations du réservoir ( $\mathcal{R}$ fluctue et polarise $\$$ ) et de la self-réaction ( $\$$ fluctue et polarise $\mathcal{R}$ qui réagit en retour sur $\$$ ) sont identifiées en suivant une méthode générale exposée dans un précédent article à propos de l'étude des processus radiatifs (contributions des fluctuations du vide et de la réaction de rayonnement). Les parties hamiltonienne et non hamiltonienne de l'évolution de $S$ sont explicitées et reliées aux fonctions de corrélation et polarisabilités de $S$ et $\mathcal{R}$. La limite où $S$ est un système quasi-classique est étudiée. On démontre que l'évolution de la fonction de distribution de l'énergie de $S$ est décrite par une équation de Fokker-Planck dont les termes de dérive et de diffusion sont respectivement associés à la self-réaction et aux fluctuations du réservoir. Ces résultats généraux sont enfin appliqués au problème de l'émission spontanée d'un grand moment cinétique (modèle de Dicke pour la superradiance).
\end{abstract}

\begin{abstract}
The physical processes responsible for the evolution of a small system $\delta$ weakly coupled to a large reservoir $\mathcal{R}$ are analysed through the coupled Heisenberg equations of the problem. The respective contributions of reservoir fluctuations ( $\mathcal{R}$ fluctuates and polarizes $S$ ) and self-reaction ( $\delta$ fluctuates and polarizes $\mathcal{R}$ which reacts back on $\$$ ) are identified with a general method developed in a previous paper dealing with radiative processes (contributions of vacuum fluctuations and radiation reaction). The Hamiltonian and non Hamiltonian parts of the evolution of $S$ are made explicit and related to correlation functions and polarizabilities of $S$ and $R$. The limit where $S$ is a quasi-classical system is investigated, and the evolution of the energy distribution function of $S$ is shown to be described by a Fokker-Planck equation, the drift and diffusion terms of which are respectively associated with self-reaction and reservoir fluctuations. These results are finally applied to the problem of the spontaneous emission of a large angular momentum (Dicke's model of superradiance).
\end{abstract}

\section{Introduction.}

In a previous paper [1], referred to as I in the following, we have considered the problem of the physical interpretation of radiative processes (radiative corrections such as the Lamb-shift or the spin anomaly $g-2$, spontaneous emission rates). It is generally considered [2-5] that, in quantum radiation theory, there exists an indetermination in the separation of the respective effects of "vacuum fluctuations " (interaction of the electron with the quantized vacuum field) and "radiation reaction » (interaction of the electron with its own field). We have shown in $I$ that such an indetermination can be removed by imposing to the corresponding two rates of variation to be Hermitian (this is necessary if we want them to have a physical meaning), and we have thus obtained results in complete agreement with the usual pictures associated with the two types of physical processes. 
Actually, the problem considered in I is very general and can be set for any small system $\mathcal{S}$ (generalizing the atom) coupled to a large reservoir $\mathcal{R}$ (generalizing the field). Is the evolution of $S$ due to the "reservoir fluctuations " acting upon $\mathcal{S}$, or should we invoke a "self-reaction ", $\mathcal{S}$ perturbing $\mathcal{R}$ which reacts back on $\mathcal{S}$ ? In this paper, we would like to discuss such a problem, to derive some general results which were just given in I without demonstration, and to give some new physical insights obtained by considering the limit where $S$ is a quasi-classical system.

In this introduction, we shall first follow the same procedure as in I for identifying the respective contributions of vacuum fluctuations and self-reaction. This will allow us to summarize the method given in I and to introduce our notations. We start from the total Hamiltonian $H$ of the combined system

$$
H=H_{\mathrm{S}}+H_{\mathrm{R}}+V,
$$

where $H_{\mathrm{S}}\left(H_{\mathrm{R}}\right)$ is the free system (reservoir) Hamiltonian, and $V$ the coupling which can always be written as

$$
V=-g \sum_{i} R_{i} S_{i}
$$

$g$ being a coupling constant (analogous to the electric charge in electrodynamics) and $S_{i}\left(R_{i}\right)$ system (reservoir) Hermitian operators. The rate of variation of an arbitrary system observable $G\left(G=G^{+}\right)$is given by Heisenberg equations, and the contribution of the coupling $V$ to this rate can be written as

where

$$
\left(\frac{\mathrm{d} G}{\mathrm{~d} t}\right)_{\text {coupling }}=-\frac{i g}{\hbar} \sum_{i}\left[R_{i}(t) S_{i}(t), G(t)\right]=g \sum_{i} N_{i}(t) R_{i}(t)
$$

$$
N_{i}(\dot{t})=-\frac{i}{\hbar}\left[S_{i}(t), G(t)\right]
$$

is an observable of the system $\mathcal{S}\left(N_{i}=N_{i}^{+}\right)$. The respective contributions of reservoir fluctuations and selfreaction are then obtained by replacing in $(1.3) R_{i}(t)$ by

where

$$
R_{i}(t)=R_{i}^{\mathrm{f}}(t)+R_{i}^{\mathrm{s}}(t)
$$

$$
R_{i}^{\mathrm{f}}(t)=\mathrm{e}^{i\left(t-t_{0}\right) H_{\mathrm{R}}\left(t_{0}\right) / h} R_{i}\left(t_{0}\right) \mathrm{e}^{-i\left(t-t_{0}\right) H_{\mathrm{R}}\left(t_{0}\right) / h}
$$

is the solution, to order 0 in $g$, of the Heisenberg equation for $R_{i}$, corresponding to a free evolution between the initial time $t_{0}$ and $t$ (analogous to the free field of electrodynamics), and $R_{i}^{\mathrm{s}}(t)$ the solution to order 1 and higher in $g$ (analogous to the source field in electrodynamics). But we are then faced with the following problem. In (1.3), $N_{i}(t)$ and $R_{i}(t)$ are commuting system and reservoir operators which can be taken in any order, so that (1.3) can be written as

$$
g \lambda \sum_{i} N_{i}(t) R_{i}(t)+g(1-\lambda) \sum_{i} R_{i}(t) N_{i}(t)
$$

with $\lambda$ arbitrary. When $R_{i}(t)$ is replaced by $(1.5)$ in (1.7), we get the following two rates of variation respectively due to reservoir fluctuations and self reaction

$$
\left\{\begin{array}{l}
\left(\frac{\mathrm{d} G}{\mathrm{~d} t}\right)_{\mathrm{rf}}=g \lambda \sum_{i} N_{i}(t) R_{i}^{\mathrm{f}}(t)+g(1-\lambda) \sum_{i} R_{i}^{\mathrm{f}}(t) N_{i}(t) \\
\left(\frac{\mathrm{d} G}{\mathrm{~d} t}\right)_{\mathrm{sr}}=g \lambda \sum_{i} N_{i}(t) R_{i}^{\mathrm{s}}(t)+g(1-\lambda) \sum_{i} R_{i}^{\mathrm{s}}(t) N_{i}(t) .
\end{array}\right.
$$

The problem is that $R_{i}^{\mathrm{f}}(t)$ and $R_{i}^{\mathrm{s}}(t)$ do not commute separately with $N_{i}(t)$, as their sum does. $\left(\frac{\mathrm{d} G}{\mathrm{~d} t}\right)_{\mathrm{rf}}$ and $\left(\frac{\mathrm{d} G}{\mathrm{~d} t}\right)_{\mathrm{sr}}$ seem therefore to depend on an arbitrary parameter $\lambda[2-5]$.

We have removed in $I$ this apparent indetermination by the following argument. $G$ being a physical observable, represented by a Hermitian operator, we want to split the total rate $\mathrm{d} G / \mathrm{d} t$, which is also Hermitian, into two distinct rates associated with two distinct physical processes, reservoir fluctuations and self-reaction. These two rates should separately have a physical meaning, and consequently should be separately Hermitian. This condition imposes $\lambda=1 / 2$ in (1.8) $\left(^{1}\right)$ and, consequently, the completely symmetrical order in (1.7). Using

( $\left.{ }^{1}\right)$ Note that $\lambda$ is implicitly taken real in (1.7), (1.8) and(1.9) [6]. Such a choice can be actually justified by time reversal symmetry arguments [see Appendix C]. 
the expression (1.4) of $N_{i}(t)$, we thus get

$$
\left\{\begin{array}{l}
\left(\frac{\mathrm{d} G}{\mathrm{~d} t}\right)_{\mathrm{rf}}=-\frac{i g}{2 \hbar} \sum_{i}\left\{\left[S_{i}(t), G(t)\right] R_{i}^{\mathrm{f}}(t)+R_{i}^{\mathrm{f}}(t)\left[S_{i}(t), G(t)\right]\right\}, \\
\left(\frac{\mathrm{d} G}{\mathrm{~d} t}\right)_{\mathrm{sr}}=-\frac{i g}{2 \hbar} \sum_{i}\left\{\left[S_{i}(t), G(t)\right] R_{i}^{\mathrm{s}}(t)+R_{i}^{\mathrm{s}}(t)\left[S_{i}(t), G(t)\right]\right\} .
\end{array}\right.
$$

The next step is to compute the average of the two rates (1.9) in the reservoir state. Such a calculation is not trivial. First, $R_{i}^{\mathrm{f}}(t)$ does not commute with $S_{i}(t)$ and $G(t)$. Secondly, the system observables $S_{i}(t)$ and $G(t)$ also operate on reservoir states since they have been " contaminated " by reservoir operators during the evolution between $t_{0}$ and $t$. When the coupling constant $g$ is small enough (more precisely when the motional narrowing condition of relaxation theory $[7,8]$ is fulfilled), a perturbative calculation of the two rates (1.9), up to order 2 in $g$, is sufficient. In I, we have just given the results of this perturbative calculation and discussed their structure.

A first motivation of this new paper is to give the details of the perturbative calculation of the reservoir averages of the two rates (1.9), and to discuss the various approximations allowing to express these averaged rates in terms of important statistical functions of the two interacting systems : correlation functions and linear susceptibilities. We also show how it is possible to decompose the various rates of variation in a Hamiltonian part, describing how the energy levels of $\mathcal{S}$ are shifted by the coupling with $\mathcal{R}$, and a non-Hamiltonian part describing dissipative effects, such as energy transfers between $S$ and $\mathcal{R}$. These calculations are presented in sections 2 and 3.

A second motivation of this paper is to present a new application of the general procedure leading to the two rates (1.9). We consider, in section 4 , the case when $S$ is a quasi-classical system. The equation giving the rate of variation of the populations of $S$ can be, in this case, transformed into a Fokker-Planck equation. We show that the separation between self-reaction effects and reservoir fluctuation effects is particularly transparent in this equation. The two effects are respectively associated with the "drift " and "diffusion " terms of the Fokker-Planck equation. Applying these results to the spontaneous emission of a large angular momentum (section 5) gives some insight to the superradiance problem.

As in the previous paper, we are concerned here with physical interpretation of equations, trying to identify in these equations what corresponds to the usual pictures of fluctuations and reaction. Whether it is possible to experimentally dissociate these two mechanisms is another interesting problem which is outside the scope of this paper.

\section{Reservoir averaged rates of variation : perturbative calculation.}

2.1 INTRODUCTION. - In this section, we first calculate a perturbative expansion of the two rates of variation $(1.9 a)$ and $(1.9 b)$, up to order 2 in $g$ [\$ 2.2 and 2.3]. We then take the average, in the reservoir state, of these two perturbative rates $[\$ 2.4]$.

We shall suppose here that, at the initial time $t_{0}$, the density operator $\rho\left(t_{0}\right)$ is factorized into a system part $\sigma_{\mathrm{S}}\left(t_{0}\right)$ and a reservoir part $\sigma_{\mathrm{R}}\left(t_{0}\right)$ :

$$
\rho\left(t_{0}\right)=\sigma_{\mathrm{S}}\left(t_{0}\right) \cdot \sigma_{\mathrm{R}}\left(t_{0}\right),
$$

In other words, we " put together » the system and the reservoir at time $t_{0}$ and let them interact. Our problem is to evaluate the subsequent rates of variation of $S$, respectively due to reservoir fluctuations and self-reaction, and averaged over the state $\sigma_{\mathrm{R}}\left(t_{0}\right)$ of the reservoir.

The more general case of a non factorized initial state is examined in appendix $C$.

2.2 Perturbative Resolution OF Heisenberg equations. - Any operator $O(t)$ evolves in time according to the Heisenberg equation

$$
\begin{aligned}
\frac{\mathrm{d}}{\mathrm{d} t} O(t) & =\frac{i}{\hbar}[H(t), O(t)] \\
& =\frac{i}{\hbar}\left[H_{\mathrm{S}}(t)+H_{\mathrm{R}}(t), O(t)\right]-\frac{i g}{\hbar} \sum_{i}\left[R_{i}(t) S_{i}(t), O(t)\right] .
\end{aligned}
$$

We want to calculate here the solution of this equation as a power series of $g$, in the case where $O$ is a system or reservoir operator. 
The first term of (2.2), which describes the free evolution of $O(t)$, is not in general proportional to $O(t)$, since several Bohr frequencies appear in the free motion of $O(t)$. It is therefore useful to introduce a basis of operators for $S$ and $\mathcal{R}$ having each a single free evolution frequency :

$$
\begin{aligned}
Q_{A B} & =|A\rangle\langle B| & \text { for operators of } \mathcal{R} \\
q_{a b} & =|a\rangle\langle b| & \text { for operators of } \delta
\end{aligned}
$$

where $|A\rangle,|B\rangle$ (resp. $|a\rangle,|b\rangle)$ are eigenstates of $H_{\mathrm{R}}$ (resp. $H_{\mathrm{S}}$ ), with energies $E_{A}, E_{B}$ (resp. $\left.\varepsilon_{a}, \varepsilon_{b}\right)$.

The Heisenberg equation for $Q_{A B}$ reads

$$
\frac{\mathrm{d}}{\mathrm{d} t} Q_{A B}(t)=i \Omega_{A B} Q_{A B}(t)-\frac{i g}{\hbar} \sum_{i} S_{i}(t)\left[R_{i}(t), Q_{A B}(t)\right],
$$

where

$$
\Omega_{A B}=\left(E_{A}-E_{B}\right) / \hbar
$$

is the unperturbed Bohr frequency associated with $|A\rangle$ and $|B\rangle$. This equation can be formally integrated to give

where

$$
Q_{A B}(t)=Q_{A B}^{\mathrm{f}}(t)+Q_{A B}^{\mathrm{s}}(t),
$$

$$
Q_{A B}^{\mathrm{f}}(t)=Q_{A B}\left(t_{0}\right) \mathrm{e}^{i \Omega_{A B}\left(t-t_{0}\right)}
$$

is the « free " part of $Q_{A B}(t)$, of order zero in the coupling constant $g$ (i.e. the value of $Q_{A B}$ if there was no coupling), and where

$$
Q_{A B}^{\mathrm{s}}(t)=-\frac{i g}{\hbar} \int_{t_{0}}^{t} \mathrm{~d} t^{\prime} \mathrm{e}^{i \Omega_{A B}\left(t-t^{\prime}\right)} \sum_{i} S_{i}\left(t^{\prime}\right)\left[R_{i}\left(t^{\prime}\right), Q_{A B}\left(t^{\prime}\right)\right]
$$

is the solution of (2.4) to order one and higher in $g$, which we shall call the " source " part of $Q_{A B}(t)$, by analogy with the atom-field problem.

The coupling constant $g$ explicitly appears in (2.8). If we want to calculate $Q_{A B}^{\mathrm{s}}(t)$ up to order one in $g$, we can replace the three operators appearing in the integral of $(2.8)$ by their free parts.

This gives

$$
Q_{A B}^{\mathrm{s}}(t)=-\frac{i g}{\hbar} \int_{t_{0}}^{t} \mathrm{~d} t^{\prime} \mathrm{e}^{i \Omega_{A B}\left(t-t^{\prime}\right)} \sum_{i} S_{i}^{\mathrm{f}}\left(t^{\prime}\right)\left[R_{i}^{\mathrm{f}}\left(t^{\prime}\right), Q_{A B}^{\mathrm{f}}\left(t^{\prime}\right)\right]+O\left(g^{2}\right)
$$

which can also be written, according to (2.7)

$$
Q_{A B}^{\mathrm{s}}(t)=-\frac{i g}{\hbar} \int_{t_{0}}^{t} \mathrm{~d} t^{\prime} \sum_{i} S_{i}^{\mathrm{f}}\left(t^{\prime}\right)\left[R_{i}^{\mathrm{f}}\left(t^{\prime}\right), Q_{A B}^{\mathrm{f}}(t)\right]+O\left(g^{2}\right) .
$$

For the small system $q_{a b}$ operators, the same algebra can be done, leading to :

with

$$
q_{a b}(t)=q_{a b}^{\mathrm{f}}(t)+q_{a b}^{\mathrm{s}}(t)
$$

$$
\left\{\begin{array}{c}
q_{a b}^{\mathrm{f}}(t)=q_{a b}\left(t_{0}\right) \mathrm{e}^{i \omega_{a b}\left(t-t_{0}\right)} \quad\left(\hbar \omega_{a b}=\varepsilon_{a}-\varepsilon_{b}\right) \\
q_{a b}^{\mathrm{s}}(t)=-\frac{i g}{\hbar} \int_{t_{0}}^{t} \mathrm{~d} t^{\prime} \sum_{i} R_{i}^{\mathrm{f}}\left(t^{\prime}\right)\left[S_{i}^{\mathrm{f}}\left(t^{\prime}\right), q_{a b}^{\mathrm{f}}(t)\right] .
\end{array}\right.
$$

These results can be generalized to any reservoir operator $R$ (resp. system operator $S$ ), using the expansion of this operator on the $Q_{A B}$ (resp. $q_{a b}$ ) basis. One gets

$$
\begin{aligned}
R(t) & =\sum_{A B} Q_{A B}(t)\langle A|R| B\rangle \\
& =R^{\mathrm{f}}(t)+R^{\mathrm{s}}(t),
\end{aligned}
$$


where the free and source parts of $R(t)$ are

$$
\left\{\begin{array}{l}
R^{\mathrm{f}}(t)=\sum_{A B} Q_{A B}^{\mathrm{f}}(t)\langle A|R| B\rangle \\
R^{\mathrm{s}}(t)=\sum_{A B} Q_{A B}^{\mathrm{s}}(t)\langle A|R| B\rangle .
\end{array}\right.
$$

The source part $R^{\mathrm{s}}(t)$ can also be written, according to (2.10)

$$
R^{\mathrm{s}}(t)=-\frac{i g}{\hbar} \int_{t_{0}}^{t} \mathrm{~d} t^{\prime} \sum_{i} S_{i}^{\mathrm{f}}\left(t^{\prime}\right)\left[R_{i}^{\mathrm{f}}\left(t^{\prime}\right), R^{\mathrm{f}}(t)\right]+O\left(g^{2}\right) .
$$

In the same way, one gets for a system operator

with

$$
S(t)=S^{\mathrm{f}}(t)+S^{\mathrm{s}}(t)
$$

$$
\left\{\begin{array}{l}
S^{\mathrm{f}}(t)=\sum_{a b} q_{a b}^{\mathrm{f}}(t)\langle a|S| b\rangle \\
S^{\mathrm{s}}(t)=-\frac{i g}{\hbar} \int_{t_{0}}^{t} \mathrm{~d} t^{\prime} \sum_{t} R_{i}^{\mathrm{f}}\left(t^{\prime}\right)\left[S_{i}^{\mathrm{f}}\left(t^{\prime}\right), S^{\mathrm{f}}(t)\right]+O\left(g^{2}\right)
\end{array}\right.
$$

Remark : Validity of this perturbative expansion.

The order of magnitude of $\left\langle Q_{A B}^{s}\right\rangle$ is, using (2.10)

$$
\left\langle Q_{A B}^{\mathrm{s}}(t)\right\rangle \simeq \frac{\left(t-t_{0}\right) \sqrt{\left\langle V^{2}\right\rangle}}{\hbar}\left\langle Q_{A B}^{\mathrm{f}}\right\rangle
$$

where $\sqrt{\left\langle V^{2}\right\rangle}$ represents the root mean square value of the coupling $V$. The following term in the perturbative expansion (2.10) of $\left\langle Q_{A B}^{\mathrm{s}}(t)\right\rangle$ would be of the order of

$$
\frac{1}{\hbar^{2}} \int_{t_{0}}^{t} \mathrm{~d} t^{\prime} \int_{t_{0}}^{t^{\prime}} \mathrm{d} t^{\prime \prime}\left\langle V\left(t^{\prime}\right) V\left(t^{\prime \prime}\right)\right\rangle\left\langle Q_{A B}^{\mathrm{f}}\left(t^{\prime}\right)\right\rangle
$$

Introducing the correlation time $\tau_{\mathrm{c}}$ of the reservoir, the integral over $t^{\prime \prime}$ can be evaluated [8]

$$
\int_{t_{0}}^{t^{\prime}} \mathrm{d} t^{\prime \prime}\left\langle V\left(t^{\prime}\right) V\left(t^{\prime \prime}\right)\right\rangle \simeq\left\{\begin{array}{lll}
\left\langle V^{2}\right\rangle\left(t^{\prime}-t_{0}\right) & \text { if } & t^{\prime}-t_{0}<\tau_{\mathrm{c}} \\
\left\langle V^{2}\right\rangle \tau_{\mathrm{c}} & \text { if } & \left.t^{\prime}-t_{0}\right\rangle \tau_{\mathrm{c}}
\end{array}\right.
$$

The neglected term (2.19) is then certainly less than

$$
\frac{\left\langle V^{2}\right\rangle \tau_{\mathrm{c}}\left(t-t_{0}\right)}{\hbar^{2}}\left\langle Q_{A B}^{\mathrm{f}}\right\rangle
$$

Our approximation is valid as long as this term is small compared to both $\left\langle Q_{A B}^{\mathrm{f}}\right\rangle$ and the first-order value (2.18) of $\left\langle Q_{A B}^{\mathrm{s}}(t)\right\rangle$.

(i) The comparison of (2.21) with (2.18) leads to

$$
\frac{\sqrt{\left\langle V^{2}\right\rangle} \tau_{\mathrm{c}}}{\hbar} \ll 1
$$

This is the well known " motional narrowing condition ": The $\mathcal{R}-\mathcal{S}$ coupling can be treated perturbatively if the effect of this coupling during a correlation time $\tau_{c}$ is small [8].

(ii) The comparison of $(2.21)$ with $\left\langle Q_{A B}^{\mathrm{f}}\right\rangle$ leads to the condition

$$
\frac{\left\langle V^{2}\right\rangle \tau_{\mathrm{c}}\left(t-t_{0}\right)}{\hbar^{2}} \ll 1
$$

If we note that $V^{2} \tau_{\mathrm{c}} / \hbar^{2}$ is the inverse of the relaxation time $T_{\mathrm{R}}$ of the system $S$ under the action of $\mathcal{R}$, we can transform (2.23) into

$$
t-t_{0} \ll T_{\mathrm{R}}
$$


The approximate expressions (2.15) and (2.17b) for the source parts of $R$ and $S$ are then valid as long as $t-t_{0}$ is small compared to the relaxation time. (Note that, as long as the motional narrowing condition (2.22) is fulfilled, the relaxation time $T_{\mathrm{R}}$ is much longer than the correlation time $\tau_{\mathrm{c}}$ )

2.3 Perturbative rates of Variation. - We can now use the results of the previous section 2.2 for evaluating the two rates of variation (1.9a) and (1.9b) up to order 2 in $g$.

We split the system operators $S_{i}(t)$ and $G(t)$ appearing in (1.9) into their free and source parts and we use the approximate expression $(2.17 \mathrm{~b})$ for the source parts. We also use the approximate expression $(2.15)$ for the source part $R_{i}^{\mathrm{s}}(t)$ of $R_{i}(t)$. This allows us, after a straightforward algebra, to get expressions for the two rates (1.9), valid up to order 2 in $g$ and containing only free operators of $\mathcal{R}$ and $S$

$$
\left\{\begin{aligned}
\left(\frac{\mathrm{d} G}{\mathrm{~d} t}\right)_{\mathrm{rf}}(t)= & -\frac{i g}{2 \hbar} \sum_{i}\left\{R_{i}^{\mathrm{f}}(t)\left[S_{i}^{\mathrm{f}}(t), G^{\mathrm{f}}(t)\right]+\left[S_{i}^{\mathrm{f}}(t), G^{\mathrm{f}}(t)\right] R_{i}^{\mathrm{f}}(t)\right\}- \\
& -\frac{g^{2}}{2 \hbar^{2}} \sum_{i j} \int_{t_{0}}^{t} \mathrm{~d} t^{\prime}\left(R_{i}^{\mathrm{f}}(t) R_{j}^{\mathrm{f}}\left(t^{\prime}\right)+R_{j}^{\mathrm{f}}\left(t^{\prime}\right) R_{i}^{\mathrm{f}}(t)\right)\left[S_{j}^{\mathrm{f}}\left(t^{\prime}\right),\left[S_{i}^{\mathrm{f}}(t), G^{\mathrm{f}}(t)\right]\right]+O\left(g^{3}\right) \\
\left(\frac{\mathrm{d} G}{\mathrm{~d} t}\right)_{\mathrm{sr}}(t)= & -\frac{g^{2}}{2 \hbar^{2}} \sum_{i j} \int_{t_{0}}^{t} \mathrm{~d} t^{\prime}\left[R_{j}^{\mathrm{f}}\left(t^{\prime}\right), R_{i}^{\mathrm{f}}(t)\right]\left(S_{j}^{\mathrm{f}}\left(t^{\prime}\right)\left[S_{i}^{\mathrm{f}}(t), G^{\mathrm{f}}(t)\right]+\left[S_{i}^{\mathrm{f}}(t), G^{\mathrm{f}}(t)\right] S_{j}^{\mathrm{f}}\left(t^{\prime}\right)\right)+O\left(g^{3}\right) .
\end{aligned}\right.
$$

we note that the self-reaction rate $(2.25 \mathrm{~b})$ is of order 2 whereas the reservoir fluctuations part contains terms of order 1 (first line of 2.25a) and 2 (second line of 2.25a). Actually, the term of order 1 appears to be the first order term of the "Langevin force " acting upon $G$. (For the exact expression of this Langevin force, see for instance $[7,9,10]$.)

The great interest of expressions (2.25) is that they can easily be averaged on the reservoir state since they only involve « free " operators. Their validity is the same as that of the expansions of $\S 2.2$. The motional narrowing condition (2.22) being fulfilled, these expressions are valid as long as $t-t_{0}$ is small compared to the relaxation time $T_{\mathrm{R}}$.

2.4 RESER VOIR AVERAGED RATES. - As explained in the introduction of this section, we take a factorized initial state (2.1). The averages of the two rates (2.25) in the reservoir state $\sigma_{\mathrm{R}}\left(t_{0}\right)$ are therefore

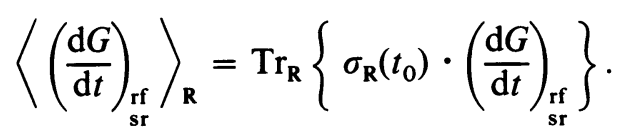

We can always suppose that the average value of $R_{i}^{\mathrm{f}}\left(t_{0}\right)$ is equal to zero, possibly by writing

$$
R_{i}^{\mathrm{f}}\left(t_{0}\right)=R_{i}^{\mathrm{f}}\left(t_{0}\right)-\left\langle R_{i}^{\mathrm{f}}\left(t_{0}\right)\right\rangle_{\mathrm{R}}+\left\langle R_{i}^{\mathrm{f}}\left(t_{0}\right)\right\rangle_{\mathrm{R}}
$$

and by reincluding $-g \sum_{i}\left\langle R_{i}^{\mathrm{f}}\left(t_{0}\right)\right\rangle_{\mathrm{R}} S_{i}(t)$ in the system Hamiltonian $H_{\mathrm{S}}$. It follows that the reservoir average of the first line of (2.25a) is equal to zero (as is expected for a Langevin force).

Since the two rates $(2.25)$ contain only free operators, their average value in the reservoir state is easily calculated. We get

$$
\left\{\begin{array}{l}
\left\langle\left(\frac{\mathrm{d} G}{\mathrm{~d} t}\right)_{\mathrm{rf}}(t)\right\rangle_{\mathrm{R}}=-\frac{g^{2}}{\hbar^{2}} \sum_{i j} \int_{t_{0}}^{t} \mathrm{~d} t^{\prime} C_{i j}^{(\mathrm{R})}\left(t, t^{\prime}\right)\left[S_{j}^{\mathrm{f}}\left(t^{\prime}\right),\left[S_{i}^{\mathrm{f}}(t), G^{\mathrm{f}}(t)\right]\right] \\
\left\langle\left(\frac{\mathrm{d} G}{\mathrm{~d} t}\right)_{\mathrm{sr}}(t)\right\rangle_{\mathrm{R}}=-\frac{i g^{2}}{2 \hbar} \sum_{i j} \int_{t_{0}}^{t} \mathrm{~d} t^{\prime} \chi_{i j}^{(\mathrm{R})}\left(t, t^{\prime}\right)\left(S_{j}^{\mathrm{f}}\left(t^{\prime}\right)\left[S_{i}^{\mathrm{f}}(t), G^{\mathrm{f}}(t)\right]+\left[S_{i}(t), G^{\mathrm{f}}(t)\right] S_{j}^{\mathrm{f}}\left(t^{\prime}\right)\right),
\end{array}\right.
$$

where we have put

$$
\begin{aligned}
& C_{i j}^{(\mathrm{R})}\left(t, t^{\prime}\right)=\frac{1}{2} \operatorname{Tr}_{\mathrm{R}}\left[\sigma_{\mathrm{R}}\left(t_{0}\right)\left(R_{i}^{\mathrm{f}}(t) R_{j}^{\mathrm{f}}\left(t^{\prime}\right)+R_{j}^{\mathrm{f}}\left(t^{\prime}\right) R_{i}^{\mathrm{f}}(t)\right)\right] \\
& \chi_{i j}^{(\mathrm{R})}\left(t, t^{\prime}\right)=\frac{i}{\hbar} \operatorname{Tr}_{\mathrm{R}}\left(\sigma_{\mathrm{R}}\left(t_{0}\right)\left[R_{i}^{\mathrm{f}}(t), R_{j}^{\mathrm{f}}\left(t^{\prime}\right)\right]\right) \theta\left(t-t^{\prime}\right),
\end{aligned}
$$

$\theta$ being the Heaviside function $(\theta(x)=1$ if $x>0, \theta(x)=0$ if $x \leqslant 0)$. The two functions $C_{i j}^{(\mathrm{R})}$ and $\chi_{i j}^{(\mathrm{R})}$ are real, and they depend only on $\tau=t-t^{\prime}$ if $\sigma_{\mathrm{R}}\left(t_{0}\right)$ commutes with $H_{\mathrm{R}}$ (other properties of these functions are listed in appendix A). $C_{i j}^{(\mathrm{R})}$ and $\chi_{i j}^{(\mathrm{R})}$ are actually two important statistical functions of the reservoir [11]. $C_{i j}^{(\mathrm{R})}$ is the symmetric correlation function, describing the dynamics of the fluctuations of $R_{i}$ and $R_{j}$ in the stationary state $\sigma_{\mathrm{R}}\left(t_{0}\right)$. 
It is a satisfactory result to see that $C_{i j}^{(\mathbf{R})}$ appears in the "reservoir fluctuations " term (2.28a). $\chi_{i j}^{(\mathbf{R})}$ is the linear susceptibility of the reservoir, determining the linear response of the averaged observable $\left\langle R_{i}(t)\right\rangle$ when the reservoir is acted upon by a perturbation proportional to $R_{j}$. It is also an interesting result to see that $\chi_{i j}^{(R)}$ characterizes the " response " of the reservoir in the self reaction rate $(2.28 \mathrm{~b})$.

The width of the two functions $C_{i j}^{(R)}$ and $\chi_{i j}^{(R)}$ is of the order of $\tau_{\mathrm{c}}$. If we restrict ourselves to time $t$ such as $t-t_{0} \gg \tau_{\mathrm{c}}$ (and $t-t_{0} \ll T_{\mathrm{R}}$ so that the perturbative calculation remains valid (see 2.24)), the expressions (2.28) can be simplified and written as

$$
\left\{\begin{array}{l}
\left\langle\left(\frac{\mathrm{d} G}{\mathrm{~d} t}\right)_{\mathrm{rf}}(t)\right\rangle_{\mathrm{R}}=-\frac{g^{2}}{\hbar^{2}} \sum_{i}\left[Y_{i}^{\mathrm{f}}(t),\left[S_{i}^{\mathrm{f}}(t), G^{\mathrm{f}}(t)\right]\right] \\
\left\langle\left(\frac{\mathrm{d} G}{\mathrm{~d} t}\right)_{\mathrm{sr}}(t)\right\rangle_{\mathrm{R}}=-\frac{i g^{2}}{2 \hbar} \sum_{i}\left(Z_{i}^{\mathrm{f}}(t)\left[S_{i}^{\mathrm{f}}(t), G^{\mathrm{f}}(t)\right]+\left[S_{i}^{\mathrm{f}}(t), G^{\mathrm{f}}(t)\right] Z_{i}^{\mathrm{f}}(t)\right),
\end{array}\right.
$$

where

$$
\left\{\begin{array}{l}
Y_{i}(t)=\sum_{a b j} q_{a b}(t)\left\langle a\left|S_{j}\right| b\right\rangle \int_{0}^{\infty} \mathrm{d} \tau C_{i j}^{(\mathbf{R})}(\tau) \mathrm{e}^{-i \omega_{a b} \tau} \\
Z_{i}(t)=\sum_{a b j} q_{a b}(t)\left\langle a\left|S_{j}\right| b\right\rangle \int_{0}^{\infty} \mathrm{d} \tau \chi_{i j}^{(\mathbf{R})}(\tau) \mathrm{e}^{-i \omega_{a b} \tau}
\end{array}\right.
$$

Note that the expressions (2.31) of the rates remain valid to order 2 in $g$, if we replace in the right member the free operator $\left[Y_{i}^{\mathrm{f}}(t),\left[S_{i}^{\mathrm{f}}(t), G^{\mathrm{f}}(t)\right]\right]$ by the reservoir average $\left\langle\left[Y_{i}(t),\left[S_{i}(t), G(t)\right]\right]\right\rangle_{\mathrm{R}}($ from $(2.11)$ and $(2.12)$, the difference is at least of order 1 in $g$ ) :

$$
\left\{\begin{array}{l}
\left\langle\left(\frac{\mathrm{d} G}{\mathrm{~d} t}\right)_{\mathrm{rf}}(t)\right\rangle_{\mathrm{R}}=-\frac{g^{2}}{\hbar^{2}} \sum_{i}\left\langle\left[Y_{i}(t),\left[S_{i}(t), G(t)\right]\right]\right\rangle_{\mathrm{R}} \\
\left\langle\left(\frac{\mathrm{d} G}{\mathrm{~d} t}\right)_{\mathrm{sr}}(t)\right\rangle_{\mathrm{R}}=-\frac{i g^{2}}{2 \hbar} \sum_{i}\left\langle Z_{i}(t)\left[S_{i}(t), G(t)\right]+\left[S_{i}(t), G(t)\right] Z_{i}(t)\right\rangle_{\mathrm{R}} .
\end{array}\right.
$$

Each rate of variation of $G(t)$ is now expressed as a function of $G(t)$ itself rather than $G^{\mathrm{f}}(t)$, and this will be more convenient for the calculations of section 3 .

Remark : Our assumption concerning the factorization (2.1) of the initial state may seem questionable. Usually, the system $S$ and the reservoir $\mathcal{R}$ are always interacting, and $S$ and $\mathcal{R}$ cannot be considered as being " put together » at some initial time $t_{0}$. It follows that, at any time $t_{0}$, the total density matrix $\rho\left(t_{0}\right)$ should rather be written

where

$$
\rho\left(t_{0}\right)=\sigma_{\mathrm{R}}\left(t_{0}\right) \cdot \sigma_{\mathrm{S}}\left(t_{0}\right)+\Delta \sigma\left(t_{0}\right),
$$

$$
\sigma_{\mathrm{R}}\left(t_{0}\right)=\operatorname{Tr}_{\mathrm{S}}\left(\rho\left(t_{0}\right)\right) ; \quad \sigma_{\mathrm{S}}\left(t_{0}\right)=\operatorname{Tr}_{\mathrm{R}}\left(\rho\left(t_{0}\right)\right),
$$

and where $\Delta \sigma\left(t_{0}\right)$ describes correlations which exist at time $t_{0}$ between $S$ and $\mathcal{R}$ as a result of their previous interaction. In the presence of such correlations, the calculations presented in this section must be modified. We show, in appendix B, that, if $\mathcal{R}$ is a large reservoir, expressions (2.33) remain valid provided that the two rates of variation are considered as " coarse grained " rates of variation, i.e. as rates of variation averaged over a time interval $\Delta t$ large compared to the correlation time $\tau_{\mathrm{c}}$, but small compared to the relaxation time $T_{\mathrm{R}}$.

\section{Effective Hamiltonian and relaxation.}

The two rates (2.33a) and (2.33b) only involve observables of the system $\delta$. Their expressions are more complicated than averaged Heisenberg rates, which are mere commutators of $G(t)$ with another $\mathcal{S}$ operator. Nevertheless, one can distinguish in these two rates a part which is of that kind, i.e., which is physically equivalent to the effect of an effective Hamiltonian $H_{\text {eff }}$. The remaining non Hamiltonian part of the evolution of $G(t)$ will simply be called relaxation.

3.1 Separation procedure. - Consider first (2.33.a). The double commutator can be developed into

$$
\left[Y_{i},\left[S_{i}, G\right]\right]=Y_{i} S_{i} G+G S_{i} Y_{i}-Y_{i} G S_{i}-S_{i} G Y_{i} .
$$

The last two terms cannot contribute to a commutator such as [ $H_{\text {eff }}, G$ ], (unless $S_{i}$ reduces to a number, but, in this case, it does not express a real coupling between $\mathcal{R}$ and $S$ ). Thus, the last two terms only contribute to relaxa- 
tion. The first two terms can be split in a unique way into the sum of a commutator and an anticommutator, so that equation (3.1) becomes :

$$
\begin{aligned}
& {\left[Y_{i},\left[S_{i}, G\right]\right]=\left[\frac{1}{2}\left(Y_{i} S_{i}-S_{i} Y_{i}\right), G\right]+\frac{1}{2}\left(Y_{i} S_{i}+S_{i} Y_{i}\right) G+\frac{1}{2} G\left(Y_{i} S_{i}+S_{i} Y_{i}\right)} \\
& -Y_{i} G S_{i}-S_{i} G Y_{i} .
\end{aligned}
$$

In the same way, the operator of $(2.33 b)$ is transformed into :

$$
\begin{aligned}
Z_{i}\left[S_{i}, G\right]+\left[S_{i}, G\right] Z_{i}=\left[\frac { 1 } { 2 } \left(Z_{i} S_{i}+\right.\right. & \left.\left.S_{i} Z_{i}\right), G\right]+ \\
& +\frac{1}{2}\left(Z_{i} S_{i}-S_{i} Z_{i}\right) G+\frac{1}{2} G\left(Z_{i} S_{i}-S_{i} Z_{i}\right)+S_{i} G Z_{i}-Z_{i} G S_{i} .
\end{aligned}
$$

We introduce the two effective Hamiltonians $\left(H_{\text {eff }}\right)_{\text {rf }}$ and $\left(H_{\text {eff }}\right)_{\text {sr }}$ corresponding to the commutator part of (3.2) and (3.3) :

$$
\begin{aligned}
& \left(H_{\mathrm{eff}}(t)\right)_{\mathrm{rf}}=\frac{i g^{2}}{2 \hbar} \sum_{i}\left[Y_{i}(t), S_{i}(t)\right] \\
& \left(H_{\mathrm{eff}}(t)\right)_{\mathrm{sr}}=-\frac{g^{2}}{4} \sum_{i}\left(Z_{i}(t) S_{i}(t)+S_{i}(t) Z_{i}(t)\right) .
\end{aligned}
$$

The two rates (2.33a) and (2.33b) are then split into two parts using (3.2) and (3.3) :

$$
\begin{aligned}
& \left\langle\left(\frac{\mathrm{d} G}{\mathrm{~d} t}\right)_{\mathrm{rf}}(t)\right\rangle_{\mathrm{R}}=\frac{i}{\hbar}\left\langle\left[\left(H_{\mathrm{eff}}(t)\right)_{\mathrm{rf}}, G(t)\right]\right\rangle_{\mathrm{R}}+ \\
& +\left(\frac{-g^{2}}{2 \hbar^{2}}\right) \sum_{i}\left\langle\left[Y_{i}(t),\left[S_{i}(t), G(t)\right]\right]+\left[S_{i}(t),\left[Y_{i}(t), G(t)\right]\right]\right\rangle_{\mathrm{R}}, \\
& \left\langle\left(\frac{\mathrm{d} G}{\mathrm{~d} t}\right)_{\mathrm{sr}}(t)\right\rangle_{\mathrm{R}}=\frac{i}{\hbar}\left\langle\left[\left(H_{\mathrm{eff}}(t)\right)_{\mathrm{sr}}, G(t)\right]\right\rangle_{\mathrm{R}}+ \\
& +\left(\frac{-i g^{2}}{4 \hbar}\right) \sum_{i}\left\langle Z_{i}(t)\left[S_{i}(t), G(t)\right]+\left[S_{i}(t), G(t)\right] Z_{i}(t)-S_{i}(t)\left[Z_{i}(t), G(t)\right]-\left[Z_{i}(t), G(t)\right] S_{i}(t)\right\rangle_{\mathrm{R}} .
\end{aligned}
$$

The first line of (3.6) (resp. (3.7)) describes the part of the evolution due to reservoir fluctuations (resp. due to self reaction) and which can be described by an effective Hamiltonian. The second line describes the non Hamiltonian part of the evolution of $G$, which we have called relaxation, caused by reservoir fluctuations (resp. self reaction). We discuss now the physical content of these equations.

3.2 EfFective Hamiltonians. - The Hamiltonian part of the evolution of $S$ is now described by $H_{\mathrm{s}}(t)+$ $\left(H_{\mathrm{eff}}(t)\right)_{\mathrm{rf}}+\left(H_{\mathrm{eff}}(t)\right)_{\mathrm{sr}}$. We calculate in this section the modification of the energy spectrum of $\delta$ due to the two effective Hamiltonians.

We have introduced above the unperturbed energy levels $|a\rangle$ of $\delta$ as the eigenstates of $H_{\mathbf{S}}\left(t_{0}\right)$. The energy shifts $\left(\delta E_{a}\right)_{\mathrm{rf}}$ and $\left(\delta E_{a}\right)_{\mathrm{sr}}$ produced respectively by reservoir fluctuations and self reaction are therefore

$$
\begin{aligned}
& \left(\delta E_{a}\right)_{\mathrm{rf}}=\left\langle a\left|\left(H_{\mathrm{eff}}\left(t_{0}\right)\right)_{\mathrm{rf}}\right| a\right\rangle, \\
& \left(\delta E_{a}\right)_{\mathrm{sr}}=\left\langle a\left|\left(H_{\mathrm{eff}}\left(t_{0}\right)\right)_{\mathrm{sr}}\right| a\right\rangle .
\end{aligned}
$$

Using formula (3.4), $\left(\delta E_{a}\right)_{\mathrm{rf}}$ becomes :

$$
\left(\delta E_{a}\right)_{\mathrm{rf}}=\frac{i g^{2}}{2 \hbar} \sum_{i}\left\langle a\left|\left[Y_{i}\left(t_{0}\right), S_{i}\left(t_{0}\right)\right]\right| a\right\rangle .
$$

Noting that $S_{i}\left(t_{0}\right)$ is identical to $S_{i}^{\mathrm{f}}\left(t_{0}\right)$ and that $Y_{i}\left(t_{0}\right)$ can be transformed into

$$
Y_{i}\left(t_{0}\right)=\sum_{j} \int_{0}^{\infty} \mathrm{d} \tau C_{i j}^{(\mathbf{R})}(\tau) S_{j}^{\mathrm{f}}\left(t_{0}-\tau\right),
$$


and introducing the susceptibility of the system $S$ in the state $|a\rangle$

we transform $\left(\delta E_{a}\right)_{\mathrm{rf}}$ into :

$$
\chi_{i j}^{(\mathrm{S}, \mathrm{a})}(\tau)=\frac{i}{\hbar}\left\langle a\left|\left[S_{i}^{\mathrm{f}}\left(t_{0}\right), S_{j}^{\mathrm{f}}\left(t_{0}-\tau\right)\right]\right| a\right\rangle \theta(\tau)
$$

$$
\left(\delta E_{a}\right)_{\mathrm{rf}}=\frac{-g^{2}}{2} \sum_{i j} \int_{-\infty}^{+\infty} C_{i j}^{(\mathbf{R})}(\tau) \chi_{i j}^{(\mathbf{S}, \mathrm{a})}(\tau) \mathrm{d} \tau
$$

i.e., as a sum of time averaged products of susceptibilities of the system $S$ by correlation functions of reservoir observables [12]. This expression has a simple physical meaning : the fluctuations of the reservoir observables polarize $S$ and produce an additional motion of $S_{i}(t)$ given by

$$
\delta S_{i}(t)=g \int_{-\infty}^{+\infty} \chi_{i j}^{(\mathrm{S}, \mathrm{a})}(\tau) R_{j}(t-\tau) \mathrm{d} \tau
$$

The corresponding polarization energy is

$$
-\frac{1}{2} g \sum_{i} R_{i}(t) . \delta S_{i}(t)
$$

as for an atom in an electric field which gets a polarization energy $-\frac{1}{2} \chi E^{2}=-\frac{1}{2} E . \delta D$ for an induced dipole $\delta D=\chi E$. Of course, equations (3.14) and (3.15) are only qualitative, since they do not take care of the operator nature of the quantities $R_{i}$ and $\delta S_{i}$. But they explain physically the structure of the exact equation (3.13). Using the symmetry properties (A.4) and (A.22) of $C$ and $\chi$ (see appendix A), one finds that only the reactive part $\chi_{i j}^{\prime(\mathbf{S}, \mathbf{a})}$ contributes to $(3.13)$ :

$$
\left(\delta E_{a}\right)_{\mathrm{rf}}=\frac{-g^{2}}{2} \sum_{i j} \int_{-\infty}^{+\infty} C_{i j}^{(\mathrm{R})}(\tau) \chi_{i j}^{(\mathrm{S}, a)}(\tau) \mathrm{d} \tau
$$

Only the reactive part of the susceptibility contributes to the polarization energy of $\delta$.

From the expression (3.5) of $\left(H_{\mathrm{eff}}\right)_{\mathrm{sr}}$, and following steps similar to those which have led us from (3.8) to (3.16), we find that the shift of the energy level $|a\rangle$ produced by self reaction is :

$$
\left(\delta E_{a}\right)_{\mathrm{sr}}=\frac{-g^{2}}{2} \sum_{i j} \int_{-\infty}^{+\infty} \chi_{i j}^{(\mathrm{R})}(\tau) C_{i j}^{(\mathrm{S}, \mathrm{a})}(\tau) \mathrm{d} \tau
$$

where $C_{i j}^{(S, a)}(\tau)$ is the symmetric correlation function of $S_{i}$ and $S_{j}$ for the system $\delta$ in the state $|a\rangle$ :

$$
C_{i j}^{(\mathrm{S}, \mathrm{a})}(\tau)=\frac{1}{2}\left\langle a\left|S_{i}^{\mathrm{f}}\left(t_{0}\right) S_{j}^{\mathrm{f}}\left(t_{0}-\tau\right)+S_{j}^{\mathrm{f}}\left(t_{0}-\tau\right) S_{i}^{\mathrm{f}}\left(t_{0}\right)\right| a\right\rangle .
$$

In the same way as in (3.16), only the reactive part of the susceptibility of $\mathcal{R}$ contributes to $\left(\delta E_{a}\right)_{\mathrm{sr}}$. Physically, it represents the polarization energy of the reservoir $\mathcal{R}$ due to the motion of the system $\delta$.

Note that the physical interpretation of both $\left(\delta E_{a}\right)_{\mathrm{rf}}$ and $\left(\delta E_{a}\right)_{\mathrm{sr}}$ is in complete agreement with the physical pictures associated with their basic origin, reservoir fluctuations and self reaction.

3.3 RELAXATION RATES OF ENERGY. - As an illustration of the relaxation processes produced by both reservoir fluctuations and self reaction, we compute the variation rates of the mean energy of $S$ initially in the state $|a\rangle$. So, we replace in (3.6) and (3.7) the observable $G$ by the Hamiltonian $H_{\mathrm{S}}$ of $S$ and we take the diagonal matrix element of both members in the state $|a\rangle$. To order two in $g$, all the operators appearing in the right side of these equations can be considered as free operators. In this approximation, the commutators of the first lines of (3.6) and (3.7) reduce to :

$$
\left\langle a\left|\left[H_{\mathrm{eff}}^{\mathrm{f}}, H_{\mathrm{S}}^{\mathrm{f}}\right]\right| a\right\rangle=0
$$

since $|a\rangle$ is an eigenstate of $H_{\mathrm{S}}^{\mathrm{f}}$. The rates (3.6) and (3.7) reduce to their relaxation parts, where we can replace $\left[S_{i}^{\mathrm{f}}(t), H_{\mathrm{S}}^{\mathrm{f}}(t)\right]$ by $i \hbar \frac{\mathrm{d}}{\mathrm{d} t} S_{i}^{\mathrm{f}}(t)$, and similarly for $Y_{i}^{\mathrm{f}}(t)$ and $Z_{i}^{\mathrm{f}}(t)$. 
We get :

$$
\begin{gathered}
\left\langle\left(\frac{\mathrm{d} H_{\mathrm{S}}}{\mathrm{d} t}\right)_{\mathrm{rf}}(t)\right\rangle=\frac{-i g^{2}}{2 \hbar} \sum_{i}\left\langle a\left|\left[Y_{i}^{\mathrm{f}}(t), \frac{\mathrm{d}}{\mathrm{d} t} S_{i}^{\mathrm{f}}(t)\right]+\left[S_{i}^{\mathrm{f}}(t), \frac{\mathrm{d}}{\mathrm{d} t} Y_{i}^{\mathrm{f}}(t)\right]\right| a\right\rangle \\
\left\langle\left(\frac{\mathrm{d} H_{\mathrm{S}}}{\mathrm{d} t}\right)_{\mathrm{sr}}(t)\right\rangle=\frac{g^{2}}{4} \sum_{i}\left\langle a\left|Z_{i}^{\mathrm{f}}(t) \cdot \frac{\mathrm{d}}{\mathrm{d} t} S_{i}^{\mathrm{f}}(t)+\frac{\mathrm{d}}{\mathrm{d} t} S_{i}^{\mathrm{f}}(t) \cdot Z_{i}^{\mathrm{f}}(t)-S_{i}^{\mathrm{f}}(t) \cdot \frac{\mathrm{d}}{\mathrm{d} t} Z_{i}^{\mathrm{f}}(t)-\frac{\mathrm{d}}{\mathrm{d} t} Z_{i}^{\mathrm{f}}(t) \cdot S_{i}^{\mathrm{f}}(t)\right| a\right\rangle .
\end{gathered}
$$

Replacing in (2.32a) and (2.32b) $q_{a b}(t)$ by $q_{a b}^{\mathrm{f}}(t)$, one expresses $Y_{i}^{\mathrm{f}}(t)$ and $Z_{i}^{\mathrm{f}}(t)$ as a function of $S_{j}^{\mathrm{f}}(t-\tau)$. Since $\left\langle a\left|S_{i}^{\mathrm{f}}(t) S_{j}^{\mathrm{f}}(t-\tau)\right| a\right\rangle$ is independent of $t$, the time derivatives on $S_{i}(t)$ can be transferred with a minus sign to $S_{j}(t-\tau)$, and written there as $\mathrm{d} / \mathrm{d} \tau$. We get in this way :

$$
\begin{aligned}
& \left\langle\left(\frac{\mathrm{d} H_{\mathrm{S}}}{\mathrm{d} t}\right)_{\mathrm{rf}}(t)\right\rangle=\frac{-i g^{2}}{\hbar} \sum_{i j} \int_{0}^{\infty} \mathrm{d} \tau C_{i j}^{(\mathrm{R})}(\tau) \frac{\mathrm{d}}{\mathrm{d} \tau}\left\langle a\left|\left[S_{j}^{\mathrm{f}}(t-\tau), S_{i}^{\mathrm{f}}(t)\right]\right| a\right\rangle \\
& \left\langle\left(\frac{\mathrm{d} H_{\mathrm{S}}}{\mathrm{d} t}\right)_{\mathrm{sr}}(t)\right\rangle=\frac{g^{2}}{2} \sum_{i j} \int_{0}^{\infty} \mathrm{d} \tau \chi_{i j}^{(\mathrm{R})}(\tau) \frac{\mathrm{d}}{\mathrm{d} \tau}\left\langle a\left|S_{j}^{\mathrm{f}}(t-\tau) S_{i}^{\mathrm{f}}(t)+S_{i}^{\mathrm{f}}(t) S_{j}^{\mathrm{f}}(t-\tau)\right| a\right\rangle .
\end{aligned}
$$

We recognize in (3.22) the dissipative part $i \chi_{i j}^{\prime(\mathbf{S}, \mathrm{a})}(\tau)$ of the susceptibility of the system $\delta$ in the state $|a\rangle$ (see formule (A.21) of Appendix A)

$$
i \chi_{i j}^{\prime \prime(\mathrm{S}, \mathrm{a})}(\tau)=\frac{i}{2 \hbar}\left\langle a\left|\left[S_{i}^{\mathrm{f}}(t), S_{j}^{\mathrm{f}}(t-\tau)\right]\right| a\right\rangle
$$

and in (3.23) the corresponding symmetric correlation function $C_{i j}^{(\mathbf{S}, \mathbf{a})}(\tau)$, so that

$$
\begin{aligned}
& \left\langle\left(\frac{\mathrm{d} H_{\mathrm{s}}}{\mathrm{d} t}\right)_{\mathrm{rf}}(t)\right\rangle=2 g^{2} \sum_{i j} \int_{0}^{\infty} \mathrm{d} \tau C_{i j}^{(\mathbf{R})}(\tau) \frac{\mathrm{d}}{\mathrm{d} \tau} i \quad \chi_{i j}^{\prime(\mathbf{S}, \mathbf{a})}(\tau) \\
& \left\langle\left(\frac{\mathrm{d} H_{\mathrm{S}}}{\mathrm{d} t}\right)_{\mathrm{sr}}(t)\right\rangle=g^{2} \sum_{i j} \int_{0}^{\infty} \mathrm{d} \tau \chi_{i j}^{(\mathbf{R})}(\tau) \frac{\mathrm{d}}{\mathrm{d} \tau} C_{i j}^{(\mathbf{S}, \mathrm{a})}(\tau) .
\end{aligned}
$$

Since $\chi_{i j}^{(\mathbf{R})}(\tau)$ is zero for $\tau<0$, the lower limit of the integral in (3.26) can be extended to $-\infty$. Using the symmetry properties (A.4) of $C_{i j}(\tau)$ and (A.22a, b) of $\chi_{i j}(\tau)$, one easily sees that only the dissipative part $\chi_{i j}^{\prime \prime(R)}(\tau)$ contributes to (3.26). Also, in (3.25), the integrand is an even function of $\tau$, so that twice the integral from 0 to $\infty$ is just the integral from $-\infty$ to $+\infty$. We finally obtain two simple formulae of the same form (after an integration by parts in (3.25)), which may also be expressed by using the Fourier transforms $\tilde{C}_{i j}(\omega)$ and $\tilde{\chi}_{i j}(\omega)$ of the corresponding functions of $\tau$ (see appendix A) :

$$
\begin{aligned}
\left\langle\left(\frac{\mathrm{d} H_{\mathrm{S}}}{\mathrm{d} t}\right)_{\mathrm{rf}}(t)\right\rangle & =-g^{2} \sum_{i j} \int_{-\infty}^{+\infty} \mathrm{d} \tau i \chi_{i j}^{\prime(\mathbf{S}, \mathbf{a})}(\tau) \frac{\mathrm{d}}{\mathrm{d} \tau} C_{i j}^{(\mathbf{R})}(\tau) \\
& =\frac{1}{2 \pi} g^{2} \sum_{i j} \int_{-\infty}^{+\infty} \mathrm{d} \omega \omega \tilde{C}_{i j}^{(\mathbf{R})}(\omega)\left(\tilde{\chi}_{i j}^{\prime(\mathbf{S}, \mathbf{a})}(\omega)\right)^{*} \\
\left\langle\left(\frac{\mathrm{d} H_{\mathrm{S}}}{\mathrm{d} t}\right)_{\mathrm{sr}}(t)\right\rangle & =g^{2} \sum_{i j} \int_{-\infty}^{+\infty} \mathrm{d} \tau i \chi_{i j}^{\prime(\mathbf{R})}(\tau) \frac{\mathrm{d}}{\mathrm{d} \tau} C_{i j}^{(\mathbf{S}, \mathbf{a})}(\tau) \\
& =-\frac{1}{2 \pi} g^{2} \sum_{i j} \int_{-\infty}^{+\infty} \mathrm{d} \omega \omega \tilde{C}_{i j}^{(\mathbf{S}, \mathbf{a})}(\omega)\left(\tilde{\chi}_{i j}^{\prime \prime(\mathbf{R})}(\omega)\right)^{*}
\end{aligned}
$$

These expressions are familiar : when a system $S$ is perturbed by a classical random field $r(t)$ through the coupling $-g r(t) S$, the energy absorption rate is [13] :

$$
\left\langle\frac{\mathrm{d} H_{\mathrm{S}}}{\mathrm{d} t}\right\rangle=\frac{1}{2 \pi} g^{2} \int_{\infty}^{+} \mathrm{d} \omega \omega \tilde{C}(\omega)\left(\tilde{\chi}^{\prime \prime}(\omega)\right)^{*}
$$

where $\tilde{C}(\omega)$ is the spectral power of $r(t)$ and $i \tilde{\chi}^{\prime \prime}(\omega)$ is the Fourier transform of the dissipative part of the sus- 
ceptibility of the system. The formula (3.27) is the extension of (3.29) for a quantum perturbation of the form (1.2). The spectral power, which, for a classical stationary random function, is just the Fourier transform of the correlation function, is replaced here by the quantum symmetric correlation function [14]. The energy relaxation rate due to reservoir fluctuations is exactly what we would expect on a physical basis : it is the power absorbed by $S$ when it is driven by reservoir fluctuations.

Conversely, the relaxation rate of energy due to self reaction (3.28) represents the power dissipated in the reservoir by the free motion of the system $S$. The damping effect of the reservoir is expressed by the dissipative part of its susceptibility.

It is clear on these examples (effective Hamiltonian, relaxation rate of energy), that the separation of $\frac{\mathrm{d} G}{\mathrm{~d} t}$ into two parts (1.9a) and (1.9b), which was based on formal considerations, leads to results which have simple physical interpretations. The corresponding pictures are consistent with the initial assignment of the process involved, reservoir fluctuations or self reaction. This can be taken as an a posteriori proof of the validity of the criterion used for this separation.

\section{Quasi-classical system $S$ coupled to a reservoir.}

4.1 Motivation. - This section is devoted to the application of the previous results to the case of a quasiclassical $\&$ system. We shall see that both self reaction and reservoir fluctuation terms take remarkably simple forms; reservoir fluctuations broaden the population distribution of $\delta$, without changing its average position, while, on the contrary, self reaction produces a drift of this distribution without deforming it.

In all this section, we assume that the following properties are fulfilled by the quasi-classical system $S[15]$ :

(i) $S$ has a single degree of freedom (or several separable ones).

(ii) The energy levels, labelled by the quantum number $n$, are locally equidistant :

$$
E_{n}-E_{n^{\prime}}=\left(n-n^{\prime}\right) \hbar \omega .
$$

Actually, (4.1) holds as soon as the difference $n-n^{\prime}$ is small compared to $n, \omega$ being the frequency of the corresponding classical motion of energy $E_{n}$.

(iii) The matrix elements $\left\langle n^{\prime}|G| n\right\rangle$ of any physical quantity $G$ decrease rapidly as a function of $n-n^{\prime}$, while being very smooth functions of $n$ (for $n-n^{\prime}$ constant).

The quasi-classical nature of $S$ introduces interesting simplifications in the general results of section 3 , mainly concerning dissipative terms. In the following, we shall then focus on the evolution of the populations of $S$. More precisely, rather than working with the population $P_{n}(t)$ of a unique level $|n\rangle$, which has no classical limit, we shall study the evolution of the average population $P(E, t)$ of an energy band of width $\delta E$ around $E$ :

$$
P(E, t)=\sum_{n} \varphi\left(E-E_{n}\right) P_{n}(t) .
$$

The width $\delta E$ of $\varphi$ is chosen small compared to $E$, but large compared to $\hbar \omega$ so that many energy levels contribute to the sum (4.2). We have thus replaced the discrete set of populations $P_{n}(t)$ by the smoothed « energy distribution function" $P(E, t)$.

4.2 Reservoir FluCtuAtion CONTRIBUtion. - The rate of variation of $P(E, t)$ under the effect of reservoir fluctuations is

$$
\left(\frac{\partial P}{\partial t}(E, t)\right)_{\mathrm{rf}}=\sum_{n} \varphi\left(E-E_{n}\right)\left(\frac{\mathrm{d} P_{n}(t)}{\mathrm{d} t}\right)_{\mathrm{rf}}
$$

We first calculate the rate $\left(\mathrm{d} P_{n} / \mathrm{d} t\right)_{\mathrm{rf}}$ from the results of section 2. Putting $G=|n\rangle\langle n|$ in equation (2.33a) and taking the average on both $\mathcal{R}$ and $S$, one gets $\left({ }^{2}\right)$

$$
\begin{aligned}
\left(\frac{\mathrm{d} P_{n}(t)}{\mathrm{d} t}\right)_{\mathrm{rf}}=\frac{-g^{2}}{\hbar^{2}}\langle n| Y S+ & S Y|n\rangle P_{n}(t)+ \\
& +\frac{g^{2}}{\hbar^{2}} \sum_{n^{\prime}}\left(\left\langle n^{\prime}|Y| n\right\rangle\left\langle n|S| n^{\prime}\right\rangle+\left\langle n^{\prime}|S| n\right\rangle\left\langle n|Y| n^{\prime}\right\rangle\right) P_{n^{\prime}}(t)
\end{aligned}
$$

(the coupling $V=-\sum_{i} g R_{i} S_{i}$ is replaced in this section by the simpler one $V=-g R S$ ). The matrix ele-

$\left({ }^{2}\right)$ We have neglected non secular couplings between populations and « coherences ». 
ment $\left\langle n^{\prime}|Y| n\right\rangle$ can be written, using (2.32a) and (4.1) :

$$
\left\langle n^{\prime}|Y| n\right\rangle=\left\langle n^{\prime}|S| n\right\rangle \int_{0}^{\infty} \mathrm{d} \tau C^{(\mathbf{R})}(\tau) \mathrm{e}^{i\left(n-n^{\prime}\right) \omega \tau}
$$

It is then convenient to put

$$
\begin{aligned}
A_{k} & =\frac{g^{2}}{\hbar^{2}}(\langle n+k|Y| n\rangle\langle n|S| n+k\rangle+\langle n+k|S| n\rangle\langle n|Y| n+k\rangle) \\
& =\frac{g^{2}}{\hbar}|\langle n|S| n+k\rangle|^{2} \tilde{C}^{(\mathbf{R})}(-k \omega) .
\end{aligned}
$$

Because of property (iii) of $\S 4.1, A_{k}$ is practically independent of $n$. Furthermore, parity properties of $\tilde{C}$ imply that

$$
A_{k} \text { real and } A_{k}=A_{-k} .
$$

Introducing a closure relation in the first line of $(4.4),\left(\frac{\mathrm{d} P_{n}}{\mathrm{~d} t}\right)_{\mathrm{rf}}$ can then be written as

$$
\left(\frac{\mathrm{d} P_{n}}{\mathrm{~d} t}\right)_{\mathrm{rf}}=-P_{n}(t)\left(\sum_{k} A_{k}\right)+\sum_{k} A_{k} P_{n+k}(t) .
$$

We now carry this last expression into $\left(\frac{\partial P}{\partial t}(E, t)\right)_{\mathrm{rf}}$ :

$$
\left(\frac{\partial P}{\mathrm{~d} t}(E, t)\right)_{\mathrm{rf}}=-\sum_{n} \sum_{k} \varphi\left(E-E_{n}\right) P_{n}(t) A_{k}+\sum_{n} \sum_{k} \varphi\left(E-E_{n}\right) P_{n+, k}(t) A_{k} .
$$

We change the index of summation $n$ in $n-k$ in the second sum to get

$$
\left(\frac{\partial P}{\partial t}(E, t)\right)_{\mathrm{rf}}=-\sum_{n} \sum_{k} \varphi\left(E-E_{n}\right) P_{n}(t) A_{k}+\sum_{n} \sum_{k} \varphi\left(E-E_{n-k}\right) P_{n}(t) A_{k} .
$$

The summations over $k$ concern both positive and negative values of $k$. Using (4.7), this can be written

$$
\left(\frac{\partial P}{\partial t}(E, t)\right)_{\mathrm{rf}}=\sum_{n} P_{n}(t) \sum_{k>0} A_{k}\left(\varphi\left(E-E_{n+k}\right)+\varphi\left(E-E_{n-k}\right)-2 \varphi\left(E-E_{n}\right)\right) .
$$

We can now use the fact that the width of the function $\varphi$ as a function of $k$ (through $E-E_{n+k}$ or $E-E_{n-k}$ ) is much larger than the width of $A_{k}$ (property (iii) of $\left.\S 4.1\right)$. The quantity $\varphi\left(E-E_{n+k}\right)+\varphi\left(E-E_{n-k}\right)-$ $2 \varphi\left(E-E_{n}\right)$ in (4.11) can then be approximated by $(\hbar k \omega)^{2} \frac{\mathrm{d}^{2} \varphi\left(E-E_{n}\right)}{\mathrm{d} E^{2}}$.

Finally, the rate $\left(\frac{\partial P}{\partial t}(E, t)\right)_{\mathrm{rf}}$ can be written as

or

$$
\left(\frac{\partial P}{\partial t}(E, t)\right)_{\mathrm{rf}}=\sum_{n} P_{n}(t) \frac{\mathrm{d}^{2} \varphi\left(E-E_{n}\right)}{\mathrm{d} E^{2}} \sum_{k>0} A_{k}(\hbar k \omega)^{2},
$$

with

$$
\left(\frac{\partial P}{\partial t}(E, t)\right)_{\mathrm{rf}}=D \frac{\partial^{2} P}{\partial E^{2}}(E, t)
$$

$$
D=\sum_{k>0} A_{k}(\hbar k \omega)^{2} .
$$

(4.13) is a very simple diffusion equation, on the energy axis, with the diffusion rate $D$. It follows that the only effect of reservoir fluctuations is a symmetrical broadening of the population distribution $P(E, t)$, while its centre remains constant. In particular, reservoir fluctuations do not change the average energy of the quasi classical system. This result can be understood in the framework of section 3 by noting that our approximations 
concerning the quasi classical nature of $S$ imply that $\chi^{\prime \prime(\mathbf{S})}$ is zero. Equation (3.27) thus gives

$$
\left\langle n\left|\left(\frac{\mathrm{d} H_{\mathrm{S}}}{\mathrm{d} t}\right)_{\mathrm{rf}}\right| n\right\rangle=0
$$

The diffusion coefficient $D$ can be interpreted by simple semiclassical arguments. Reservoir fluctuations induce a random walk of the system on the energy axis with steps multiple of $\hbar \omega$. The transition rate $\Gamma_{k}$ from level $n$ to levels $n \pm k$ is

$$
\Gamma_{k}=\frac{2 g^{2}}{\hbar^{2}}|\langle n+k|S| n\rangle|^{2} \tilde{C}(k \omega)
$$

i.e., the product of the square of the matrix element of $S$ by the spectral density $\tilde{C}(k \omega)$ of the fluctuating field created by the reservoir at the frequency $k \omega$. The diffusion coefficient $D_{k}$ associated with a random walk of step $\hbar k \omega$ is then equal to half the square of the energy step times the transition rate

$$
D_{k}=\frac{1}{2}(\hbar k \omega)^{2} \Gamma_{k} .
$$

Summing (4.17) over $k>0$ gives (4.14).

4. 3 Self REACTION CONTRIBUtion. - Self reaction terms can be calculated in the same way as reservoir fluctuations ones. One first determines the rate $\left(\frac{\mathrm{d} P_{n}}{\mathrm{~d} t}\right)_{\mathrm{sr}}$ and then carries the result into

$$
\left(\frac{\partial P}{\partial t}(E, t)\right)_{\mathrm{sr}}=\sum_{n} \varphi\left(E-E_{n}\right)\left(\frac{\mathrm{d} P_{n}(t)}{\mathrm{d} t}\right)_{\mathrm{sr}}
$$

From section 2 (see Eq. (2.33b)), one has :

$$
\begin{aligned}
\left(\frac{\mathrm{d} P_{n}(t)}{\mathrm{d} t}\right)_{\mathrm{sr}}=- & \frac{i g^{2}}{2 \hbar}\langle n|Z S-S Z| n\rangle P_{n}(t)+ \\
& +\frac{i g^{2}}{2 \hbar} \sum_{n^{\prime}} P_{n^{\prime}}(t)\left(\left\langle n^{\prime}|Z| n\right\rangle\left\langle n|S| n^{\prime}\right\rangle-\left\langle n^{\prime}|S| n\right\rangle\left\langle n|Z| n^{\prime}\right\rangle\right) .
\end{aligned}
$$

We now introduce the real coefficient $B_{k}$ :

$$
B_{k}=\frac{i g^{2}}{2 \hbar}(\langle n+k|Z| n\rangle\langle n|S| n+k\rangle-\langle n+k|S| n\rangle\langle n|Z| n+k\rangle) .
$$

Using (2.32b), $B_{k}$ can be written as

$$
B_{k}=\frac{i g^{2}}{2 \hbar}|\langle n|S| n+k\rangle|^{2}\left(\tilde{\chi}^{(\mathbf{R})}(-k \omega)-\tilde{\chi}^{(\mathbf{R})}(k \omega)\right) .
$$

Since $\tilde{\chi}^{(\mathrm{R})}(-\Omega)=\tilde{\chi}^{(\mathrm{R}) *}(\Omega)$, we get from (A.19)

$$
B_{k}=\frac{g^{2}}{\hbar}|\langle n|S| n+k\rangle|^{2} \tilde{\chi}^{\prime \prime(\mathrm{R})}(k \omega),
$$

with the following property (compare with (4.7))

$$
B_{k} \text { real and } B_{k}=-B_{-k} .
$$

As $A_{k}$ in the previous paragraph, $B_{k}$ decreases rapidly when $|k|$ increases, while being nearly independent of $n$. The rate $\left(\mathrm{d} P_{n} / \mathrm{d} t\right)_{\text {sr }}$ of $(4.19)$ can be written

$$
\left(\frac{\mathrm{d} P_{n}(t)}{\mathrm{d} t}\right)_{\mathrm{sr}}=-\left(\sum_{k} B_{k}\right) P_{n}(t)+\sum_{k} B_{k} P_{n+k}(t) .
$$


We now put this rate into $\left(\frac{\partial P}{\partial t}(E, t)\right)_{\mathrm{sr}}$

$$
\left(\frac{\partial P}{\partial t}(E, t)\right)_{\mathrm{sr}}=-\sum_{n} \sum_{k} \varphi\left(E-E_{n}\right) B_{k} P_{n}+\sum_{n} \sum_{k} \varphi\left(E-E_{n-k}\right) B_{k} P_{n} .
$$

The first term is zero since, with (4.23)

$$
\sum_{k} B_{k}=0
$$

$\left(\frac{\partial P}{\partial t}(E, t)\right)_{\mathrm{sr}}$ then becomes :

$$
\begin{aligned}
\left(\frac{\partial P}{\partial t}(E, t)\right)_{\mathrm{sr}} & =\sum_{n} \sum_{k} \varphi\left(E-E_{n-k}\right) B_{k} P_{n} \\
& =\sum_{n} \sum_{k>0}\left(\varphi\left(E-E_{n-k}\right)-\varphi\left(E-E_{n+k}\right)\right) B_{k} P_{n} .
\end{aligned}
$$

Because of the presence of $B_{k}$ in (4.27), only small values of $k$ contribute and the quantity $\varphi\left(E-E_{n-k}\right)-$ $\varphi\left(E-E_{n+k}\right)$ can be approximated by

$$
2 \hbar k \omega \frac{\mathrm{d} \varphi\left(E-E_{n}\right)}{\mathrm{d} E} .
$$

We finally get

or

$$
\left(\frac{\partial P}{\partial t}(E, t)\right)_{\mathrm{sr}}=\left(\sum_{n} \frac{\mathrm{d} \varphi\left(E-E_{n}\right)}{\mathrm{d} E} P_{n}\right)\left(\sum_{k>0} 2 \hbar k \omega B_{k}\right)
$$

$$
\left(\frac{\partial P}{\partial t}(E, t)\right)_{\mathrm{sr}}=K \cdot \frac{\partial P}{\partial E}(E, t)
$$

with

$$
K=\sum_{k>0} 2 \hbar k \omega B_{k}=\sum_{k} \hbar k \omega B_{k} .
$$

Equation (4.29) is a propagation equation : the energy distribution function drifts along the energy axis with the "speed " $K$. Note that $-K$ is equal to the energy lost per unit of time by the system $\delta$ in the state $n$,. because of self reaction (see Eq. (3.28))

$$
\begin{aligned}
\left\langle\left(\frac{\mathrm{d} H_{\mathrm{S}}}{\mathrm{d} t}\right)_{\mathrm{sr}}\right\rangle & =-\frac{g^{2}}{2 \pi} \int \mathrm{d} \Omega \Omega \tilde{\chi}^{\prime \prime(\mathrm{R})}(\Omega) \tilde{C}^{(\mathbf{S}, n)}(\Omega) \\
& =-\frac{g^{2}}{2} \int_{\mathrm{d}} \mathrm{d} \Omega \Omega \tilde{\chi}^{\prime \prime(\mathbf{R})}(\Omega) \sum_{k}|\langle n|S| n+k\rangle|^{2}(\delta(\Omega-k \omega)+\delta(\Omega+k \omega)) \\
& =-\sum_{k} \hbar k \omega B_{k} .
\end{aligned}
$$

If we combine the results of this section, we find that the energy distribution function of a quasi classical system obeys a Fokker-Planck type equation

$$
\frac{\partial P}{\partial t}(E, t)=K \frac{\partial P}{\partial E}(E, t)+D \frac{\partial^{2} P}{\partial E^{2}}(E, t) .
$$

The drift term $K \partial P / \partial E$ describes the emission or absorption of energy by the system, resulting from self reaction effects, while the diffusion term $D \partial^{2} P / \partial E^{2}$ describes the broadening of the distribution function caused by reservoir fluctuations.

\section{Application to the spontaneous emission of a large angular momentum.}

5.1 InTRODUCTiON. - This last section is devoted to the application of the previous results to a specific example : we study the emission of energy by a large angular momentum coupled to the quantized radiation field in its vacuum state, which plays the rôle of a large reservoir. 
The angular momentum is supposed to be put in a static magnetic field $B_{0}$ so that its energy levels are equally spaced with a splitting $\hbar \omega_{0}$, where $\omega_{0}=-\gamma B_{0}$ is the Larmor frequency ( $\gamma$ gyromagnetic ratio). We thus have

$$
H_{\mathrm{S}}=\omega_{0} J_{z}
$$

The Hamiltonian of the reservoir is simply

$$
H_{\mathbf{R}}=\sum_{\mathbf{k \varepsilon}} \hbar \omega a_{\mathbf{k \varepsilon}}^{+} a_{\mathbf{k} \varepsilon}
$$

where $a_{\mathbf{k} \varepsilon}\left(a_{\mathbf{k \varepsilon}}^{+}\right)$is the destruction (creation) operator of a photon in the mode $\mathbf{k} \varepsilon$. We take a magnetic dipole coupling between $\mathbf{J}$ and the radiation field

$$
V=-\gamma \mathbf{J} . \mathbf{B}
$$

where $\mathbf{B}$ is the magnetic field operator.

At the initial time, the angular momentum is supposed to be in its upper level $|J, m=J\rangle$.

We want to study here the contribution of reservoir fluctuations (i.e. vacuum fluctuations) and self reaction to the evolution of the system :

(i) in the early stage of the emission (i.e. : the transition $|J, J\rangle \rightarrow|J, J-1\rangle)(\S 5.2)$,

(ii) in the following regime where the populated levels $|J, m\rangle$ are such that $J-m$ is large compared to 1 . The angular momentum can then be treated as a quasi classical system ( $\S 5.3)$ and the results of $\S 4$ apply.

Our principal motivation for studying this problem lies in the fact that the spontaneous emission of a large angular momentum is a simple model [16] for describing the superradiance of $2 J$ two level atoms put initially in their excited state. We shall see that vacuum fluctuations and self reaction equally contribute to the initial stage of superradiance, but that self reaction becomes predominant in the following part of the process.

5.2 EARLY STAGE OF THE EMISSION PROCESS. - If the angular momentum is put into its upper level $|J, J\rangle$, vacuum fluctuations and self reaction will both induce transitions towards $|J, J-1\rangle$. The easiest way to compare their contributions is to study the two rates $\left\langle\left(\frac{\mathrm{d} H_{\mathrm{s}}}{\mathrm{d} t}\right)_{\mathrm{rf}}\right\rangle$ and $\left\langle\left(\frac{\mathrm{d} H_{\mathrm{s}}}{\mathrm{d} t}\right)_{\mathrm{sr}}\right\rangle$ for $\sigma_{\mathrm{s}}=|J, J\rangle\langle J, J|$.

We shall not give here the explicit calculations of the statistical functions $\chi^{\prime \prime(\mathbf{R})}, C^{(\mathbf{R})}$ and $\chi^{\prime \prime(\mathbf{S})}, C^{(\mathbf{S})}$ involved in these rates; a similar algebra has already been performed in I. We just indicate the final result

$$
\left\langle\left(\frac{\mathrm{d} H_{\mathrm{s}}}{\mathrm{d} t}\right)_{\mathrm{rf}}\right\rangle=\left\langle\left(\frac{\mathrm{d} H_{\mathrm{s}}}{\mathrm{d} t}\right)_{\mathrm{sr}}\right\rangle=\hbar \omega_{0} \frac{\Gamma}{2}
$$

where we have put

$$
\Gamma=\frac{\hbar \omega_{0}^{3} \gamma^{2} J}{3 \pi \varepsilon_{0} c^{5}}
$$

We therefore find that vacuum fluctuations and self reaction equally contribute to the emission of energy in the early stage of superradiance (half the total rate for each process).

Such a result also appears in the analysis of superradiance based on a Bloch vector $(\langle\mathbf{J}\rangle)$ approach [16], where the emission process is described in terms of a pendulum starting from its metastable (upwards) equilibrium position. Without fluctuations, the pendulum would remain indefinitely in this position. Actually, the quantum fluctuations of atomic dipole moment, and those of the quantum vacuum field play an essential rôle in the initial stage of the process by removing the pendulum from its metastable position; they introduce a small «tipping angle» $\theta$. The correct equation of motion for $\langle\mathbf{J}\rangle$ is obtained if one takes [16]

$$
\left\langle\theta^{2}\right\rangle=\frac{2}{J} \text {. }
$$

The tipping angle $\theta_{\mathrm{sr}}$ due to self reaction can be obtained simply by remarking that, since $\mathbf{J}$ is a quantum operator, $\left\langle J_{x}^{2}+J_{y}^{2}\right\rangle$ is non zero. This gives

$$
\left\langle\theta_{\mathrm{sr}}^{2}\right\rangle=\frac{\left\langle J_{x}^{2}+J_{y}^{2}\right\rangle}{\left\langle J_{z}^{2}\right\rangle}=\frac{1}{J} .
$$

Since the power radiated by the pendulum is obviously proportional to $\theta^{2}$, one finds that the emission rate due to self reaction is only half of the total rate (compare (5.6) and (5.7)). The remaining part is due to vacuum fluctuations. 
Remark : This result is actually not specific of the previous simple example. It also occurs for the spontaneous emission of any excited atomic level by electric dipole coupling : the contributions of vacuum fluctuations and self reaction for transitions towards lower levels are the same and equal to half the total emission rate [1]. This explains the well known following result : if one tries to calculate the spontaneous emission rate of a two level atom by coupling it to a classical random field having an energy $\hbar \omega / 2$ per mode, one gets only half the emission rate. This is simply due to the fact that one calculates in this way only the vacuum fluctuations rate. The missing emission rate is simply that due to self reaction.

5.3 FOLLOWING STAGE OF THE EMISSION PROCESS. - When the populated levels $|J, m\rangle$ are such as $J-m \gg 1$, the results obtained for a quasi classical system $(\S 4)$ can be applied : the levels are equally spaced and a given level $|J, m\rangle$ is only coupled to $|J, m-1\rangle$ and $|J, m+1\rangle$ by the coupling $V(5.3)$. Furthermore, matrix elements of $V$ vary very smoothly with $m$ (as $\sqrt{J(J+1)-m(m+1)})$. One therefore gets a Fokker-Planck type equation (4.32) for the evolution of the energy distribution function $P(E, t)$ :

with $\left({ }^{3}\right)$

$$
\frac{\partial P}{\partial t}=K \frac{\partial P}{\partial E}+D \frac{\partial^{2} P}{\partial E^{2}}
$$

$$
\begin{aligned}
& K=\hbar \omega_{0} \Gamma \frac{J^{2}-m^{2}}{2 J}, \\
& D=\hbar \omega_{0} K .
\end{aligned}
$$

As seen in $\S 4$, the emission of energy (term $K \partial P / \partial E$ ) is entirely due to self reaction, the only effect of vacuum fluctuations being to broaden the energy distribution (term $D \partial^{2} P / \partial E^{2}$ ).

Moreover, the vacuum fluctuation term is in fact negligible in the present case; the order of magnitude of $D \partial^{2} P / \partial E^{2}$ is $(D / \delta E),(\partial P / \partial E)$, where $\delta E$ is the width of $P(E, t)$, so that one gets from $(5.10)$ :

$$
D \frac{\partial^{2} P}{\partial E^{2}} \simeq \frac{\hbar \omega_{0}}{\delta E} K \frac{\partial P}{\partial E}
$$

Since the width $\delta E$ of $P(E, t)$ has been taken much larger than $\hbar \omega_{0}$ (see $\S 4.1$ ), we have

$$
D \frac{\partial^{2} P}{\partial E^{2}} \ll K \frac{\partial P}{\partial E}
$$

The evolution of the system in this later stage appears then as being mainly due to self reaction. The energy distribution function drifts as a « quasi classical probability packet » [16] downward the energy axis.

When the emission process is completed, the angular momentum ends in the lower level $|J,-J\rangle$. Vacuum fluctuation effects play again a very important rôle; they exactly balance the energy loss due to self reaction, ensuring the stability of the ground state [14].

\section{Appendix A. - Some properties of correlation functions and susceptibilities.}

We illustrate these properties, for the reservoir $\mathcal{R}$ in a stationary state defined by the density matrix $\sigma_{\mathbf{R}}\left(\sigma_{\mathrm{R}}\right.$ commutes with the Hamiltonian $H_{\mathrm{R}}$ ). Similar results also hold for the system $\delta$ in an eigenstate of $H_{\mathrm{S}}$. We use here the same definitions as in references [11] and [13] for the Fourier transforms $\tilde{C}(\omega)$ and $\tilde{\chi}(\omega)$. The $\hat{C}(\omega)$ and $\hat{\chi}(\omega)$ of reference [1] are related to $\tilde{C}$ and $\tilde{\chi}$ by :

$$
\hat{C}(\omega)=\frac{1}{2 \pi} \tilde{C}(-\omega) \quad \hat{\chi}(\omega)=\frac{1}{2 \pi} \tilde{\chi}(-\omega) .
$$

Correlation functions :

$$
C_{i j}\left(t, t^{\prime}\right)=\frac{1}{2} \operatorname{Tr}_{\mathrm{R}}\left\{\sigma_{\mathrm{R}}\left(R_{i}^{\mathrm{f}}(t) R_{j}^{\mathrm{f}}\left(t^{\prime}\right)+R_{j}^{\mathrm{f}}\left(t^{\prime}\right) R_{i}^{\mathrm{f}}(t)\right)\right\} .
$$

For a stationary state, $C_{i j}$ depends only on $\tau=t-t^{\prime}$

$$
C_{i j}(\tau)=\frac{1}{2} \operatorname{Tr}_{\mathrm{R}}\left\{\sigma_{\mathrm{R}}\left(R_{i}^{\mathrm{f}}\left(t_{0}\right) R_{j}^{\mathrm{f}}\left(t_{0}-\tau\right)+R_{j}^{\mathrm{f}}\left(t_{0}-\tau\right) R_{i}^{\mathrm{f}}\left(t_{0}\right)\right)\right\}
$$

$\left({ }^{3}\right)$ The simple relation between $D$ and $K$ is actually a consequence of the fluctuation-dissipation theorem applied to the electromagnetic field in its ground state (reservoir at zero temperature). 
$C_{i j}(\tau)$ is real. The expression (A.2) being invariant in the exchange of $(i, t) \leftrightarrow\left(j, t^{\prime}\right)$, we have the important property :

$$
C_{i j}(\tau)=C_{j i}(-\tau)
$$

Developing on the eigenstates of $H_{\mathrm{R}}$, one can write (A.2) as

$$
C_{i j}(\tau)=\frac{1}{2} \sum_{\alpha \beta}\left\langle\alpha\left|\sigma_{\mathrm{R}}\right| \alpha\right\rangle\left(\left\langle\alpha\left|R_{i}\right| \beta\right\rangle\left\langle\beta\left|R_{j}\right| \alpha\right\rangle \mathrm{e}^{i \omega_{\alpha \beta} \tau}+\text { c.c. }\right) .
$$

The Fourier transform $\tilde{C}_{i j}$ is defined by :

$$
\tilde{C}_{i j}(\omega)=\int \mathrm{d} \tau C_{i j}(\tau) \mathrm{e}^{i \omega \tau}
$$

From the reality of $C_{i j}$ it follows that

$$
\tilde{C}_{i j}(\omega)=\left(\tilde{C}_{i j}(-\omega)\right)^{*}
$$

From the property (A.3), we get

$$
\tilde{C}_{i j}(\omega)=\tilde{C}_{j i}(-\omega)=\left(\tilde{C}_{j i}(\omega)\right)^{*}
$$

Susceptibilities :

$$
\chi_{i j}\left(t, t^{\prime}\right)=\frac{i}{\hbar} \operatorname{Tr}_{\mathrm{R}}\left\{\sigma_{\mathrm{R}}\left[R_{i}^{\mathrm{f}}(t), R_{j}^{\mathrm{f}}\left(t^{\prime}\right)\right]\right\} \theta\left(t-t^{\prime}\right) .
$$

Stationarity makes $\chi_{i j}$ depend only on $\tau=t-t^{\prime}$

$$
\chi_{i j}(\tau)=\frac{i}{\hbar} \operatorname{Tr}_{\mathrm{R}}\left\{\sigma_{\mathrm{R}}\left[R_{i}^{\mathrm{f}}\left(t_{0}\right), R_{j}^{\mathrm{f}}\left(t_{0}-\tau\right)\right]\right\} \theta(\tau) .
$$

$\chi_{i j}(\tau)$ is real and is equal to zero for $\tau<0$. It is easily seen on formula (A.9) that exchanging $(i, t)$ and $\left(j, t^{\prime}\right)$ amounts to changing $\theta\left(t-t^{\prime}\right)$ into $-\theta\left(t-t^{\prime}\right)$. Using the fact that $\theta\left(t-t^{\prime}\right)+\theta\left(t^{\prime}-t\right)=1$, we obtain the relation

$$
\chi_{i j}(\tau)-\chi_{j i}(-\tau)=\frac{i}{\hbar}\left\langle\left[R_{i}^{\mathrm{f}}\left(t_{0}\right), R_{j}^{\mathrm{f}}\left(t_{0}-\tau\right)\right]\right\rangle
$$

valid for $\tau$ positive or negative. Using a development on the eigenstates of $H_{\mathrm{R}}$, one can put (A.10) in the following form :

$$
\chi_{i j}(\tau)=\sum_{\alpha \beta}\left\langle\alpha\left|\sigma_{\mathrm{R}}\right| \alpha\right\rangle\left(\frac{i}{\hbar}\left\langle\alpha\left|R_{i}\right| \beta\right\rangle\left\langle\beta\left|R_{j}\right| \alpha\right\rangle \mathrm{e}^{i \omega_{\alpha \beta} \tau}+\text { c.c. }\right) \theta(\tau)
$$

Its Fourier transform is given by :

$$
\begin{aligned}
& \tilde{\chi}_{i j}(\omega)=\int \mathrm{d} \tau \chi_{i j}(\tau) \mathrm{e}^{i \omega \tau}= \\
&=\sum_{\alpha \beta} \frac{-\pi}{\hbar}\left\langle\alpha\left|\sigma_{\mathrm{R}}\right| \alpha\right\rangle\left\{\left\langle\alpha\left|R_{i}\right| \beta\right\rangle\left\langle\beta\left|R_{j}\right| \alpha\right\rangle\left(\frac{1}{\pi} \mathfrak{d} \frac{1}{\omega+\omega_{\alpha \beta}}-i \delta\left(\omega+\omega_{\alpha \beta}\right)\right)\right. \\
&\left.\quad-\left\langle\alpha\left|R_{j}\right| \beta\right\rangle\left\langle\beta\left|R_{i}\right| \alpha\right\rangle\left(\frac{1}{\pi} \mathfrak{T} \frac{1}{\omega-\omega_{\alpha \beta}}-i \delta\left(\omega-\omega_{\alpha \beta}\right)\right)\right\} .
\end{aligned}
$$

The reality of $\chi_{i j}(\tau)$ implies that

$$
\tilde{\chi}_{i j}(\omega)=\left(\tilde{\chi}_{i j}(-\omega)\right)^{*}
$$


One distinguishes the reactive nart $\chi_{i j}^{\prime}(\omega)$, corresponding to the principal parts, and the dissipative part $\chi_{i j}^{\prime \prime}(\omega)$ involving the $\delta$ functions :

$$
\begin{aligned}
& \tilde{\chi}_{i j}(\omega)=\tilde{\chi}_{i j}^{\prime}(\omega)+i \tilde{\chi}_{i j}^{\prime \prime}(\omega) \\
& \tilde{\chi}_{i j}^{\prime}(\omega)=\sum_{\alpha \beta} \frac{-1}{\hbar}\left\langle\alpha\left|\sigma_{\mathrm{R}}\right| \alpha\right\rangle\left\{\left\langle\alpha\left|R_{i}\right| \beta\right\rangle\left\langle\beta\left|R_{j}\right| \alpha\right\rangle \mathfrak{S}\left(\frac{1}{\omega+\omega_{\alpha \beta}}\right)-\right. \\
& \left.-\left\langle\alpha\left|R_{j}\right| \beta\right\rangle\left\langle\beta\left|R_{i}\right| \alpha\right\rangle \mathfrak{T}\left(\frac{1}{\omega-\omega_{\alpha \beta}}\right)\right\} \\
& \tilde{\chi}_{i j}^{\prime \prime}(\omega)=\sum_{\alpha \beta} \frac{\pi}{\hbar}\left\langle\alpha\left|\sigma_{\mathrm{R}}\right| \alpha\right\rangle\left\{\left\langle\alpha\left|R_{i}\right| \beta\right\rangle\left\langle\beta\left|R_{j}\right| \alpha\right\rangle \delta\left(\omega+\omega_{\alpha \beta}\right)-\left\langle\alpha\left|R_{j}\right| \beta\right\rangle\left\langle\beta\left|R_{i}\right| \alpha\right\rangle \delta\left(\omega-\omega_{\alpha \beta}\right)\right\} .
\end{aligned}
$$

For a symmetric susceptibility $(i=j), \tilde{\chi}_{i i}^{\prime}$ and $\tilde{\chi}_{i i}^{\prime \prime}$ correspond to the real and imaginary parts of $\tilde{\chi}_{i i}$. This property is not true in general, but is replaced by the properties

$$
\begin{aligned}
& \tilde{\chi}_{i j}^{\prime}(\omega)=\left(\tilde{\chi}_{j i}^{\prime}(\omega)\right)^{*} \\
& \tilde{\chi}_{i j}^{\prime \prime}(\omega)=\left(\tilde{\chi}_{j i}^{\prime \prime}(\omega)\right)^{*} .
\end{aligned}
$$

From (A.15) and (A.17), one easily gets

$$
\tilde{\chi}_{i j}^{\prime \prime}(\omega)=\frac{i}{2}\left(\left(\tilde{\chi}_{j i}(\omega)\right)^{*}-\tilde{\chi}_{i j}(\omega)\right)
$$

and then

$$
\begin{aligned}
\chi_{i j}^{\prime \prime}(\tau) & =\frac{i}{2}\left(\chi_{j i}(-\tau)-\chi_{i j}(\tau)\right) \\
& =\frac{1}{2 \hbar}\left\langle\left[R_{i}^{\mathrm{f}}\left(t_{0}\right), R_{j}^{\mathrm{f}}\left(t_{0}-\tau\right)\right]\right\rangle
\end{aligned}
$$

by using (A.10).

The Fourier transform of (A.18a and b) gives the symmetry relations :

$$
\begin{aligned}
& \chi_{i j}^{\prime}(\tau)=\chi_{j i}^{\prime}(-\tau) \\
& \chi_{i j}^{\prime \prime}(\tau)=-\chi_{j i}^{\prime \prime}(-\tau) .
\end{aligned}
$$

\section{Appendix B. - Coarse grained rates of variation.}

The reservoir averaged rates of variation (2.28) have been calculated in $\S 2$ with the assumption of a factorized initial state (2.1). We consider in this appendix the more general case of a non factorized initial state (2.34) (see remark at the end of $\S 2$ ).

The fact that $\mathcal{R}$ is a large reservoir has interesting consequences [8] on the reservoir operator $\sigma_{\mathrm{R}}\left(t_{0}\right)$ appearing in (2.34) and on the correlation term $\Delta \sigma\left(t_{0}\right)$ :

(i) The state of the large reservoir is not perturbed by the small system. We can therefore neglect the $t_{0}$ dependence of $\sigma_{\mathrm{R}}\left(t_{0}\right)$, which will be simply written $\sigma_{\mathrm{R}}$.

(ii) The correlations $\Delta \sigma\left(t_{0}\right)$, which exist between $S$ and $\mathcal{R}$ at time $t_{0}$, contribute to the future evolution of $S$, but only within a correlation time $\tau_{\mathrm{c}}$, i.e. in the interval $\left[t_{0}, t_{0}+\tau_{\mathrm{c}}\right]$. After this interval, the effect of the correlations $\Delta \sigma\left(t_{0}\right)$ washes out.

We consider now the average of the two rates (2.25) in the state (2.34). The contribution of the factorized part of (2.34), $\sigma_{\mathrm{S}}\left(t_{0}\right) \sigma_{\mathrm{R}}$, is the same as in (2.28). Since it is given by an integral from $t_{0}$ to $t$ involving statistical functions of the reservoir $C^{(\mathrm{R})}$ and $\chi^{(\mathrm{R})}$, this contribution vanishes for $t=t_{0}$, increases over an interval $\tau_{\mathrm{c}}$ and then saturates (for $t-t_{0} \gg \tau_{\mathrm{c}}$ ) to the value (2.31). On the other hand, the contribution of the correlation part $\Delta \sigma$ of (2.34) is non zero only for $t-t_{0} \leqslant \tau_{\mathrm{c}}$ because of the point (ii) mentioned above. It follows that, if we average the rates of variation over a time interval $\left[t_{0}, t_{0}+\Delta t\right]$ such that

$$
\tau_{\mathrm{c}} \ll \Delta t \ll T_{\mathbf{R}},
$$

we can ignore the contribution of $\Delta \sigma$, and replace the contribution of $\sigma_{\mathrm{S}}\left(t_{0}\right) \sigma_{\mathrm{R}}$ by its asymptotic value (2.31); the relative error associated with these two approximations is of the order of $\tau_{\mathrm{c}} / \Delta t$. With such a " coarse grained average " at time $t_{0}$, we implicitly renounce to a detailed knowledge of the dynamics of $S$ on a time scale $\tau_{\mathrm{c}}$. 
Actually, the coarse grained average must be done on the part of the rate of variation which is due to the coupling (and not to the system Hamiltonian $H_{\mathrm{S}}$ ). Mathematically, it is therefore more convenient to use a set of operators $\tilde{q}_{a b}(t)$ having no free evolution and defined by :

$$
q_{a b}(t)=\tilde{q}_{a b}(t) \mathrm{e}^{i \omega_{a b} t}
$$

The coarse grained rate of variation is thus given by :

$$
\left\langle\frac{\Delta \tilde{q}_{a b}}{\Delta t}\right\rangle\left(t_{0}\right)=\frac{1}{\Delta t} \int_{t_{0}}^{t_{0}+\Delta t} \mathrm{~d} t\left\langle\frac{\mathrm{d} \tilde{q}_{a b}}{\mathrm{~d} t}(t)\right\rangle
$$

where $\frac{\mathrm{d} \tilde{q}_{a b}}{\mathrm{~d} t}(t)$ is deduced from (2.25). We then make the two previously mentioned approximations, and replace $\left\langle\frac{\mathrm{d} \tilde{q}_{a b}}{\mathrm{~d} t}(t)\right\rangle$ by its asymptotic value deduced from (2.31). After a straightforward algebra, one gets for example for the reservoir fluctuation term (compare with 2.33) :

$$
\left\langle\frac{\Delta \tilde{q}_{a b}}{\Delta t}\right\rangle\left(t_{0}\right)=\frac{-g^{2}}{\hbar^{2}}\left(\sum_{i}\left\langle\left[Y_{i}\left(t_{0}\right),\left[S_{i}\left(t_{0}\right), \tilde{q}_{a b}\left(t_{0}\right)\right]\right]\right\rangle\right)_{\mathrm{SEC}}
$$

where the index «SEC » means that, in the expansion of the atomic operator $\left[Y_{i}\left(t_{0}\right),\left[S_{i}\left(t_{0}\right), \tilde{q}_{a b}\left(t_{0}\right)\right]\right]$ in the basis $\tilde{q}_{c d}\left(t_{0}\right)$, only the secular terms $\tilde{q}_{c d}\left(t_{0}\right)$ with $\left|\omega_{c d}-\omega_{a b}\right| \ll \Delta t^{-1}$ are kept. This is due to the fact that non secular couplings $\left|\omega_{c d}-\omega_{a b}\right| \geqslant \Delta t^{-1}$ are washed out in the integral (B.3) by a factor :

$$
\frac{1}{\Delta t} \int_{t_{0}}^{t_{0}+\Delta t} \mathrm{~d} t \mathrm{e}^{i\left(\omega_{a b}-\omega_{c d}\right) t} \lesssim \frac{1}{\Delta t\left|\omega_{a b}-\omega_{c d}\right|}
$$

Equations (2.33a) and (2.33b) can therefore be generalized to any time $t_{0}$, provided that they are understood as secular equations for coarse grained rates of variation.

Appendix C. - Time reversal properties of the two rates of variation due to self reaction and reservoir fluctuations. In the splitting $(1.7)$ of $\sum_{i} R_{i}(t) N_{i}(t)$, we have implicitly supposed $\lambda$ real, so that hermiticity of reservoir fluctuation terms (1.8a) and self reaction ones (1.8b) implies $\lambda=1 / 2$. Actually, one could consider taking. $\lambda$ complex, in which case hermiticity would only require $\lambda+\lambda^{*}=1$, i.e., $\lambda=1 / 2+i \kappa(\kappa$ real $)$. But it appears that $\kappa$ must be zero if we want that the two rates of variation respectively due to vacuum fluctuations and self reaction behave separately as " good velocities ».

Consider a physical quantity $G$ having a given signature in time reversal. If $G$ is even, $\mathrm{d} G / \mathrm{d} t$ is odd. It seems reasonable to impose separately the same property to reservoir fluctuation terms and self reaction ones. Actually, since we calculate only coarse grained rates of variation at time $t_{0},\left(\frac{\Delta G}{\Delta t}\right)_{\mathrm{rf}}\left(t_{0}\right)$ and $\left(\frac{\Delta G}{\Delta t}\right)_{\mathrm{sf}}\left(t_{0}\right)$ (see appendix B), it is sufficient to consider time reversal around $t_{0}$. Furthermore, the total interaction Hamiltonian is even, so that $R_{i}$ and $S_{i}$ can be chosen with a well defined parity. It then follows that $R_{i}^{\mathrm{f}}$ and $R_{i}^{\mathrm{s}}$ have the same parity as $R_{i}$ in time reversal around $t_{0}$ (see $(2.7)$ and $\left.(2.8)\right)\left({ }^{4}\right)$. Putting this result in $(1.8)$, one easily sees that $(\mathrm{d} G / \mathrm{d} t)_{\mathrm{rf}}(t)$ and $(\mathrm{d} G / \mathrm{d} t)_{\mathrm{sf}}(t)$ have the parity opposite to that of $G$ in time reversal around $t_{0}$ if and only if $\kappa$ is equal to zero. Such a property remains valid for the coarse grained rates $\left(\frac{\Delta G}{\Delta t}\right)_{\mathrm{rf}}\left(t_{0}\right)$ and $\left(\frac{\Delta G}{\Delta t}\right)_{\mathrm{sf}}\left(t_{0}\right)(\operatorname{see}(\mathrm{B} .3))$.

We have therefore shown that $\lambda$ has to be real in order that $\left(\frac{\Delta G}{\Delta t}\right)_{\mathrm{rf}}\left(t_{0}\right)$ and $\left(\frac{\Delta G}{\Delta t}\right)_{\mathrm{sr}}\left(t_{0}\right)$ behave as " good velocities ", i.e., that they have the opposite signature of $G$ in a time reversal around $t_{0}$.

$\left({ }^{4}\right)$ In equation (2.19b) of [1], giving the source field $\left(R_{\mathrm{i}}^{\mathrm{s}}\right)$ in terms of the atomic velocity $\pi / M$ and acceleration $\dot{\pi} / M$, $t-t_{0}$ is implicitly supposed to be positive. In the general case, the acceleration term is found to be multiplied by the sign of $\left(t-t_{0}\right)$, ensuring the good time reversal symmetry for $R_{\mathrm{i}}^{\mathrm{s}}$. 


\section{References}

[1] Dalibard, J., Dupont-Roc, J. and Cohen-Tannoudji, C., J. Physique 43 (1982) 1617.

[2] Senitzky, I. R., Phys. Rev. Lett. 31 (1973) 955.

[3] Milonni, P. W., ACkerhalt, J. R. and Smith, W. A., Phys. Rev. Lett. 31 (1973) 958.

[4] Milonni, P. W. and Smith, W. A., Phys. Rev. A 11 (1975) 814.

[5] Milonni, P. W., Phys. Rep. 25 (1976) 1.

[6] This implicit assumption in [1] has been pointed out to us by S. EPSTEIN (Private Communication).

[7] Agarwal, G. S., Springer Tracts in Modern Physics, Volume 70 (Springer Verlag, Berlin) 1974.

Agarwal, G. S., Phys. Rev. A 10 (1974) 717.

[8] Cohen-Tannoudi, C., in Frontiers in Laser Spectroscopy, Volume 1, les Houches 1975, Session XXVII, edited by BaLIaN, R., HaROCHE, S. and Liberman, S. (North Holland, Amsterdam) 1977, p. 3.

[9] Grabert, H., Z. Phys. B 26 (1977) 79.
[10] Cohen-Tannoudj, C., Cours du Collège de France 1978-79, Paris (unpublished).

[11] Martin, P., in Many Body Physics, les Houches 1967, edited by DE WiTT, C. and Balian, R. (Gordon and Breach, New York) 1968, p. 39.

[12] Duplantier, B., Thèse 3e cycle, 1978, Paris (unpublished). Expressions of this type can also be found in the context of quantum electrodynamics in Huang, K., Phys. Rev. 101 (1956) 1173.

[13] Landau, L. and Lifchitz, E. M., Statistical Physics (Pergamon Press) 1958, section 124.

[14] Fain, V. M., Sov. Phys. J.E.T.P. 23 (1966) 882.

FAIN, V. M. and Khanin, Y. I., Quantum Electronics (M.I.T. Press, Cambridge) 1969.

[15] LANDAU, L. and Lifchitz, E. M., Quantum Mechanics (Pergamon Press) 1958, section 46.

[16] Gross, M. and Haroche, S., Phys. Rep. 93 (1982) 301 and references therein. 